\title{
TSFDC: A Trading Strategy Based on Forecasting Directional Changes
}

\author{
Amer M. Bakhach ${ }^{\mathrm{a}}$, Edward P. K. Tsang ${ }^{\mathrm{a}}$, and V L Raju Chinthalapati ${ }^{\mathrm{b}}$ \\ ${ }^{a}$ Centre for Computational Finance and Economic Agents, University of Essex, Colchester, UK. \\ abakhaa,edward (@essex.ac.uk) \\ ${ }^{b}$ Dept. Accounting and Finance, University of Greenwich, Greenwich, UK. v.l.r.chinthalapati@gre.ac.uk
}

\begin{abstract}
SUMMARY
Directional Change (DC) is a technique to summarize price movements in a financial market. According to the DC concept, data is sampled only when the magnitude of price change is significant according to the investor. In this paper, we develop a contrarian trading strategy named TSFDC. TSFDC is based on a forecasting model which aims to predict the change of the direction of market's trend under the DC context. We examine the profitability, risk and risk-adjusted return of TSFDC in the FX market using eight currency pairs. We argue that TSFDC outperforms another DC-based trading strategy.
\end{abstract}

Keywords - Algorithmic trading; directional change; FX trading.

\section{INTRODUCTION}

Directional Change (DC) is an approach to summarize prices movement (Ao and Tsang [1]). Under the DC framework, the market is simplified as alternating uptrend and downtrend. A trend is identified as a change in market price larger than, or equal to, a specific threshold. This threshold, named theta, is set by the observer and usually expressed as percentage. A trend ends whenever a price change of the same threshold, theta, is observed in the inverse direction. For example, a market downtrend ends when we observe a price rise of magnitude theta; in this case we say that the market changes its direction to an uptrend. Similarly, a market's uptrend ends when we observe a price drop of magnitude theta. Many studies (e.g. [2] [3] [4] [5]) have reported that the DC framework is helpful in studying the foreign exchange (FX) markets. However, developing trading strategies based on the DC framework still in its early stages.

The literature encompasses enormous amount of trading strategies. Many of these trading strategies are based on forecasting models. Some of these forecasting models have the traditional objective of predicting the change of the direction of market's trend (e.g. [6] [7] [8] [9] [10]). Recently, Bakhach et al., [11] proposed a forecasting model, under the DC context, which aims to answer the question of whether the current trend will continue for a specific percentage before the trend ends. They also showed that, in some cases, the accuracy of their proposed forecasting model was over $80 \%$. However, they did not present any trading strategy. The establishment of such trading strategy is important in the sense of giving some empirical guarantee that the proposed forecasting method can be used in real-world [12].

In this paper we present a novel trading strategy named TSFDC. TSFDC relies on the forecasting model introduced by Bakhach et al., [11] to decide when to initiate a trade. We examine the performance of TSFDC in the FX market using eight currency pairs. We evaluate the profitability, risk and risk-adjusted performance of TSFDC. We compare the performance of TSFDC to another DC-based trading strategy.

The paper continues as follows: Section 2 describes the concept of Directional Changes. Section 3 provides a brief summary of the forecasting model introduced in Bakhach et al., [11]. We present TSFDC and its trading rules in Section 4. We discuss the selection and preparation of the datasets 
and the employed evaluation metrics in Section 5. The details of the experiments, conducted to evaluate the performance of TSFDC, are provided in Section 6. Section 7 reports and discusses the results of these experiments. We compare our trading strategy with another DC-based strategy in Section 8. Finally, we summarize the major findings of this paper in Section 9.

\section{DIRECTIONAL CHANGES}

\subsection{The DC Framework: The main concept}

In this section, we explain how market prices are summarized based on the DC concept ( [1] [13]). Directional change (DC) is an approach to summarize price changes. Under the DC framework, the market is represented as alternating uptrends and downtrends. The basic idea is that the magnitude of price changes during an uptrend, or a downtrend, must be at least equal to a specific threshold theta. Here, theta is a percentage that the observer considers substantial. One observer may consider $0.1 \%$ an important change, while another observer may consider a price's change of $2 \%$ as important. Observers who use different thresholds will observe different DC events and trends. Any price's change less than the identified threshold will not be considered as a trend when summarizing market prices.

Let us consider a market in a downtrend. Let $P_{E X T}$ be the lowest price in this downtrend and $P_{c}$ be the current price. We say that the market switches its direction from downtrend to uptrend whenever $P_{c}$ becomes greater than $P_{E X T}$ by at least theta (where theta is the threshold predetermined by the observer; usually expressed as a percentage). Similarly, if the market is in uptrend, $P_{E X T}$ would refer to the highest price in this uptrend. We say that the market switches its direction from an uptrend to a downtrend if $P_{C}$ is lower than $P_{E X T}$ by at least theta (the threshold predetermined by the observer). The detection of a new uptrend or a new downtrend is a formalized inequality, as shown in (1).

$$
\left|\frac{P_{c}-P_{E X T}}{P_{E X T}}\right| \geq \text { theta }
$$

If (1) holds, then the time at which the market traded at $P_{E X T}$ is called an 'extreme point' (e.g. points $\mathrm{A}$ and $\mathrm{D}$ in Fig. 2), and the time at which the market trades at $P_{c}$ is called a DC confirmation point, or DCC point for short (e.g. points $A^{0.1}$ and $D^{0.1}$ in Fig. 2). Note that whilst an extreme point is the end of one trend, it is also the start of the next trend, which has an opposite direction. An extreme point is only recognized in hindsight; precisely at the DCC point. For example, in Fig. 2, at point $\mathrm{A}^{0.1}$ we confirm that point $\mathrm{A}$ is an extreme point. Similarly, in Fig. 2, at point $\mathrm{D}^{0.1}$ we confirm that point $\mathrm{D}$ is an extreme point.

Under the DC framework, a trend is dissected into a DC event and an overshoot (OS) event. A DC event starts with an extreme point and ends with a DCC point. We refer to a specific DC event by its starting point, i.e. extreme point, and its DCC point. For example, in Fig. 2 the DC event which starts at point $\mathrm{B}$ and ends at point $\mathrm{B}^{0.1}$ is denoted as $\left[\mathrm{BB}^{0.1}\right]$. An OS event starts at the DCC point and ends at the next extreme point. 


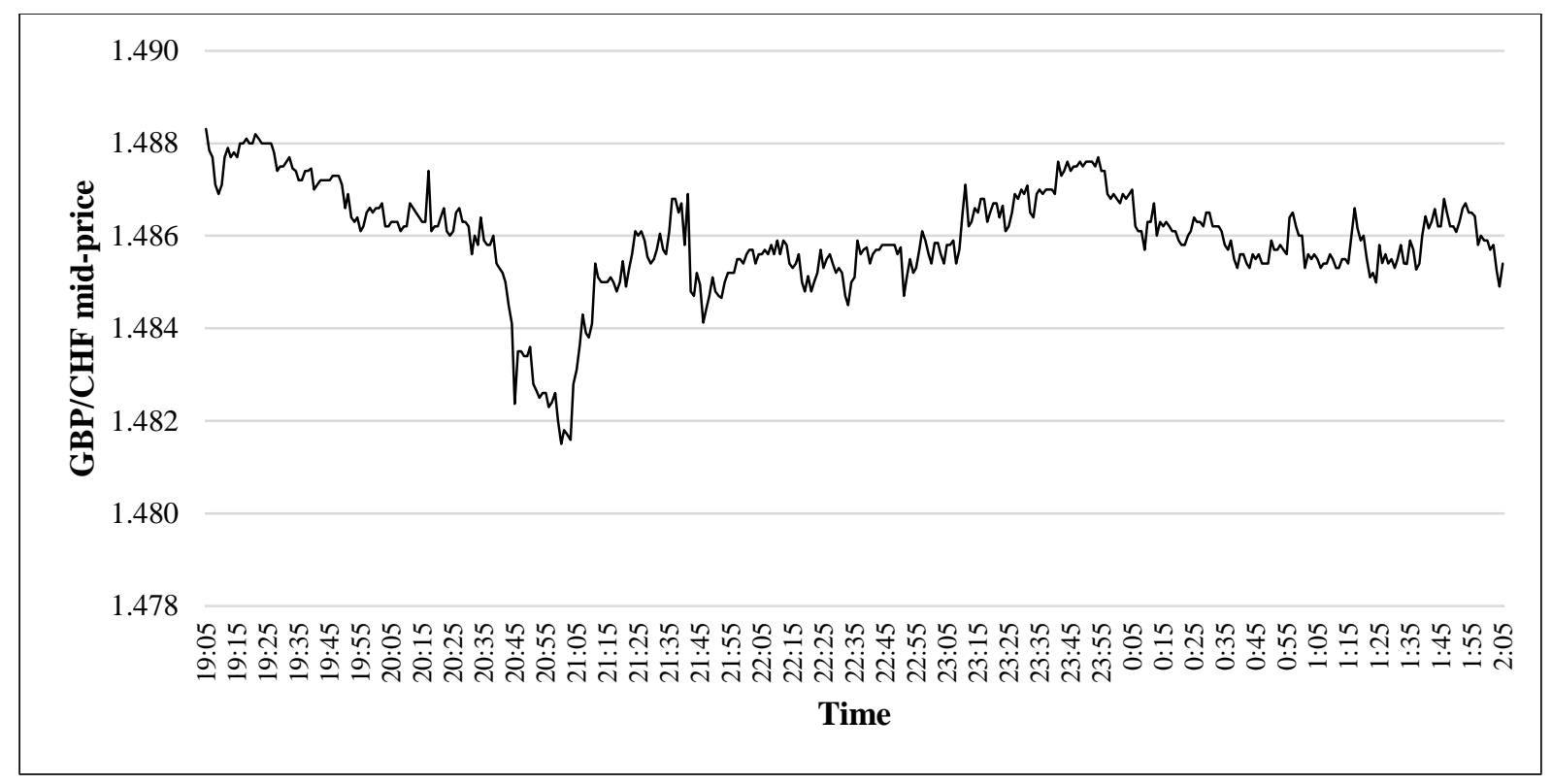

Fig. 1. GBP/CHF mid-prices sampled minute by minute from 1/1/2013 19:05 to 1/2/2013 02:05 (UK).

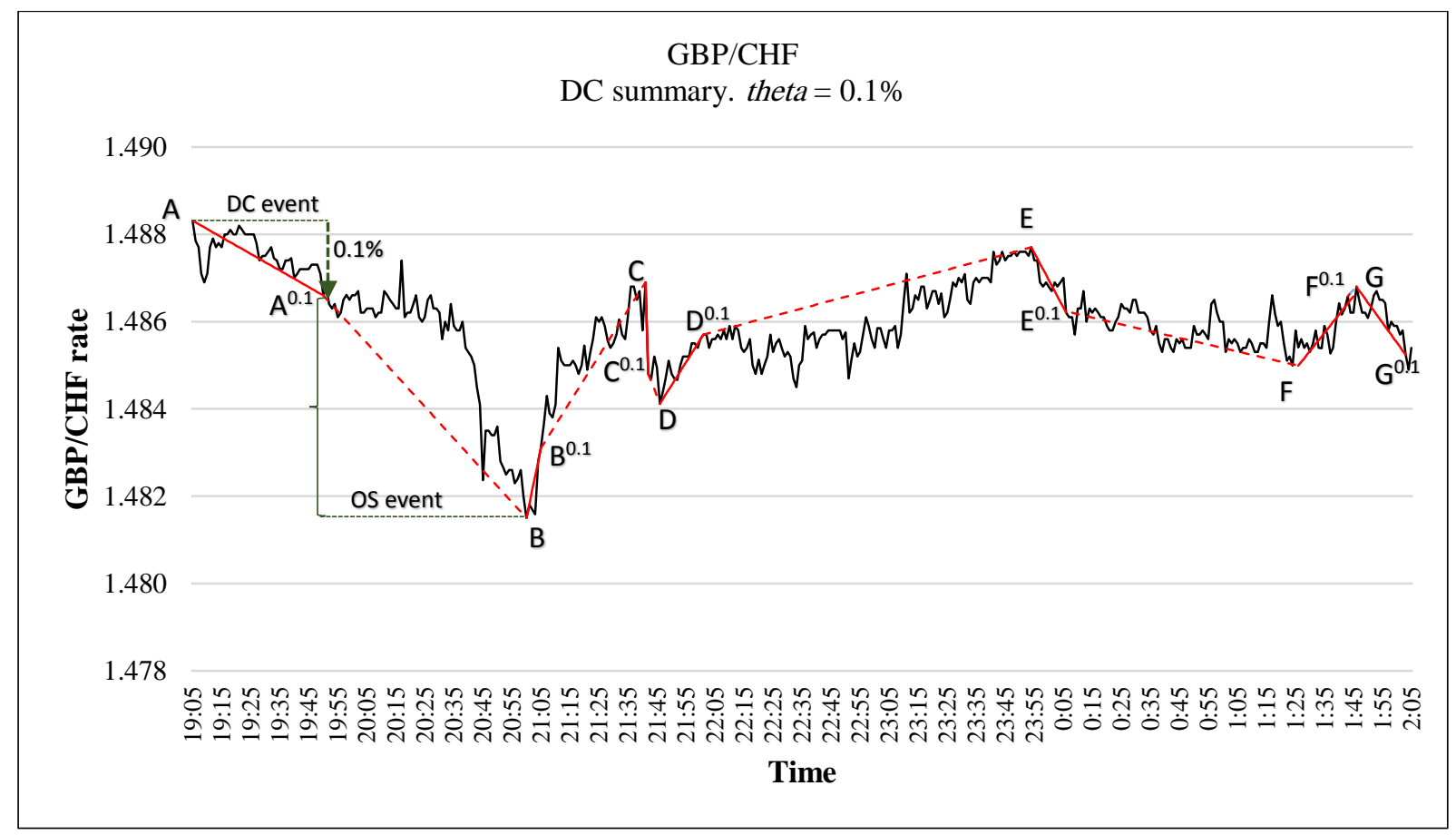

Fig. 2. An example of a DC-based summary of the price series shown in Fig. 1. Threshold theta $=0.1 \%$. The black line indicates GBP/CHF mid-prices sampled minute by minute. Solid red lines represent DC events. Dashed red lines represent OS events. Each of the points A, B, C, D, E, F, G is an extreme point. Each of the points $\mathrm{A}^{0.1}, \mathrm{~B}^{0.1}, \mathrm{C}^{0.1}, \mathrm{D}^{0.1}, \mathrm{E}^{0.1}, \mathrm{~F}^{0.1}, \mathrm{G}^{0.1}$ is a DC confirmation point (DCC point).

The DC summary of a given market is the identification of the DC and OS events, governed by the threshold theta. Fig. 2 illustrates an example of a DC summary. Note that for a given time series and a predetermined threshold, the DC summary is unique. However, we may generate multiple DC summaries for the same considered prices series by selecting multiple thresholds. The chosen threshold determines what constitutes a directional change. For example, Fig. 2 and Fig. 3 provide two distinct DC summaries, using two different thresholds, for the same prices series. If a greater threshold been chosen, then less directional changes would have been concluded between prices. For instance, in Fig. 2 the DC summary of threshold $0.1 \%$ reveals 4 downtrends and 3 uptrends. Whereas, in Fig. 3 the DC summary of threshold $0.2 \%$ detects 2 downtrends and 1 uptrend. 


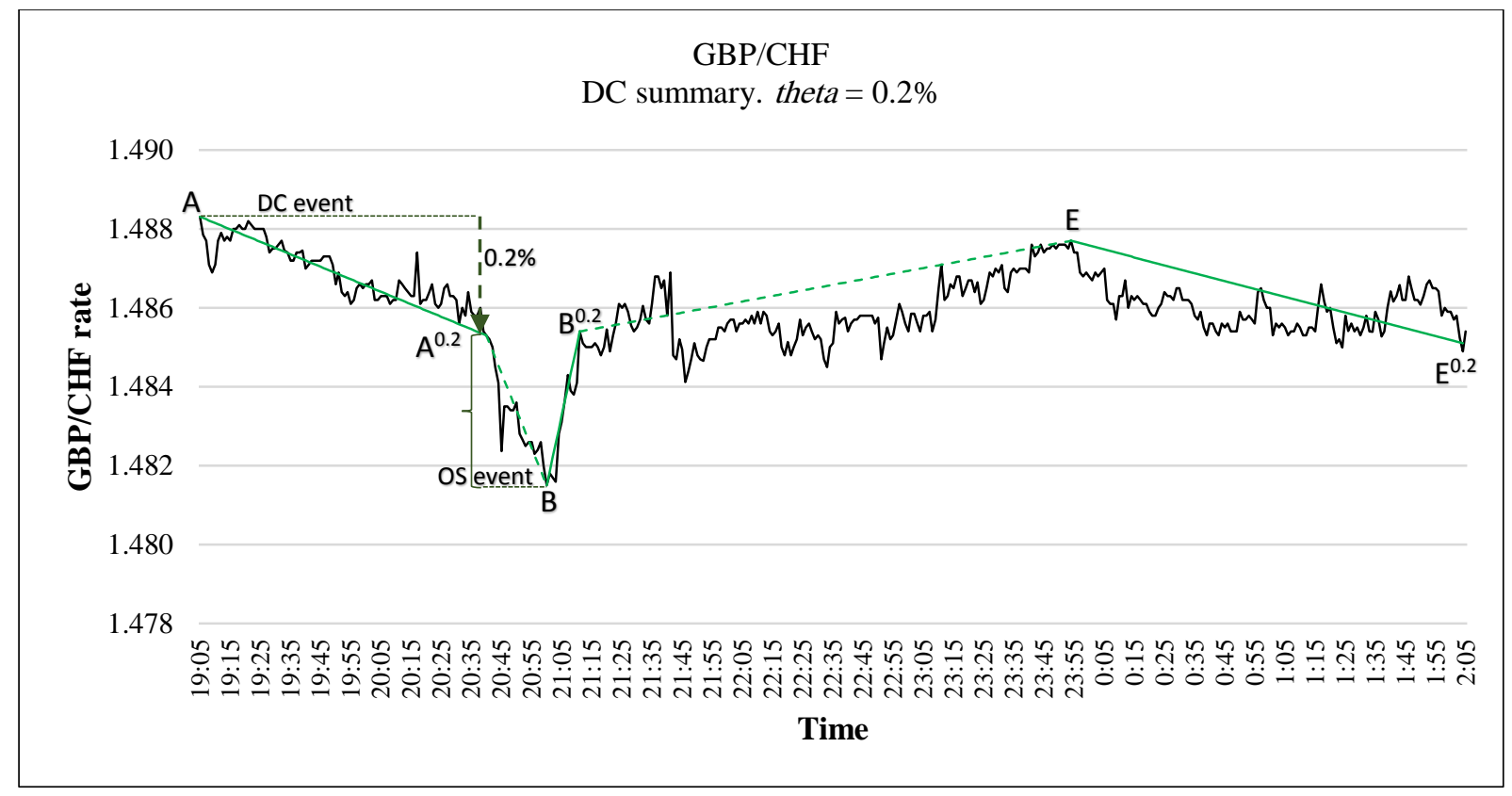

Fig. 3. An example of a DC-based summary of the price series shown in Fig. 1. theta $=0.2 \%$. The black line indicates GBP/CHF midprices. Solid green lines represent DC events. Dashed green lines represent OS events. Each of the points A, B, E is an extreme point. Each of the points $\mathrm{A}^{0.2}, \mathrm{~B}^{0.2}, \mathrm{E}^{0.2}$ is a DC confirmation point.

In this paper, we use some DC-based notations those were established by Tsang et al., [14]. Table 1 lists these notations with basic descriptions. In Table 1, if the market is in downtrend (uptrend), $P_{E X T}$ would refer to the highest (lowest) price in the overshoot period and $P_{D C C \downarrow^{*}}\left(P_{D C C \uparrow^{*}}\right)$ denotes the price required to confirm a new downtrend (uptrend) of threshold theta. In other words, in the case of DC uptrend, if $P_{C} \leq P_{D C C \downarrow} *$ then we confirm a new downward DC event. Similarly, in the case of DC downtrend, if $P_{C} \geq P_{D C C} *$ then we confirm a new upward DC event. In Table 1, $P D C C^{*}$ denotes the price required to confirm a new DC event (either uptrend or downtrend). That is:

$$
\left|\frac{P D C C^{*}-P_{E X T}}{P_{E X T}}\right| \geq \text { theta }
$$

Table 1: List of some notations used in this paper (source: Tsang et al. [14])

\begin{tabular}{|l|l|}
\hline Name / Description & Notation \\
\hline Threshold & theta \\
\hline Current price & $P_{C}$ \\
\hline $\begin{array}{l}\text { Price at extreme point: price at which one trend ends and a } \\
\text { new trend starts. }\end{array}$ & $P_{E X T}$ \\
\hline $\begin{array}{l}\text { The highest price, during an uptrend's OS event, required } \\
\text { to confirm that the market's direction has changed to } \\
\text { downtrend (i.e. to confirm a downtrend's DC event). }\end{array}$ & $P_{D C C \downarrow *}=P_{E X T} \times(1-$ theta $)$ \\
\hline $\begin{array}{l}\text { The least price, during a downtrend's OS event, required } \\
\text { to confirm that the market's direction has changed to } \\
\text { uptrend (i.e. to confirm an uptrend's DC event). }\end{array}$ & $P_{D C C \uparrow *}=P_{E X T} \times(1+$ theta $)$ \\
\hline $\begin{array}{l}P D C C^{*} \text { is the price of the theoretical directional change } \\
\text { confirmation point of the current trend. }\end{array}$ & $\begin{array}{l}P D C C^{*}=P_{D C C \downarrow *} \text { If the current trend is } \\
\text { downtrend; otherwise } P D C C^{*}=P_{D C C \uparrow *}\end{array}$ \\
\hline
\end{tabular}


The DC concept is similar to the zigzag indicator ( [15] [16]). The zigzag approach models price movement as alternating uptrend and downtrend. The price change during an uptrend or a downtrend must be at least equal to a specific threshold. The main difference between the DC approach and the zigzag indicator is that a trend, under the DC methodology, is dissected into: 1) a DC event of fixed percentage equal to the selected threshold and 2) an OS event represented by the remaining part of the trend before it reverses. This segmentation of a trend into DC and OS event, under the DC framework, has been proved to be helpful to analyse and characterize financial markets ( [2] [3] [14] [17] [18]).

\subsection{The DC Framework: A literature review}

In this section, we briefly review some studies those have reported that the DC framework has helped in analysing financial markets. For instance, in 2011, Glattfelder et al. [3] revealed new scaling laws (i.e. stylized facts), based on the DC concept, which uncover innovative facts in the FX market. The authors considered five years of tick-by-tick data for 13 exchange rates. Many of these scaling law try to model the relationship between the DC and OS events. Two examples of these scaling laws are: 1) on average, a DC event of threshold theta is followed by an OS event of same scale theta, and 2) on average, the OS event lasts about the double amount of time that it took for the DC event to complete. Fig. 4 illustrates these two scaling laws.

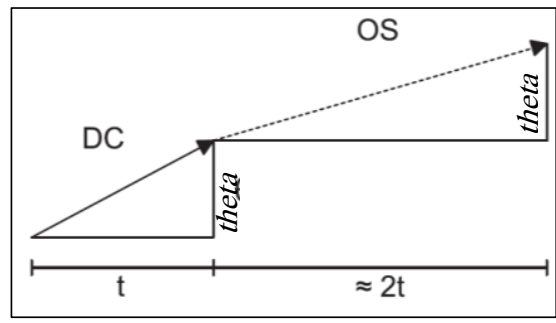

Fig. 4. An illustration of two scaling laws related to the DC and OS events reported in Glattfelder et al. [3].

In addition, in 2012, Bisig et al. [17] presented the so-called Scale of Market Quakes (SMQ) based on the DC concept. SMQ aims to quantify FX market activity during significant economic and political events declarations. For this purpose, SMQ quantifies the excess price moves during the OS event. Furthermore, in 2013, a study that deciphers FX market activity based on the DC concept was reported in Masry [4]. The introduced approach lays "the foundations for understanding how FX market activity changes as the price movement progresses" and explains how minor differences in market activities can change the price trend, under definite conditions, during the OS event. In 2014, Golub et al. [19] proposed a new way to measure the liquidity in the FX market based on the DC framework. Their new approach seeks to model market dynamic to predict stress in financial markets. They define an information theoretic measurement termed liquidity that characterises the instability of price curves during the overshoot event. They argued that the new metric can forecast stress in financial markets. They proposed that their model to quantify liquidity in the FX market can be used as an early warning system [19]. In 2017, Tsang et al. [14] introduced an approach to profiling companies and financial markets. Their methodology is based on a set of innovative indicators. These indicators are based on the DC analysis of high frequency price movements. They conclude that information obtained through DC-based analysis and from time series complement each other.

The literature also encompasses few studies those sought to develop trading strategies based on the DC framework. For instance, in 2016, Bakhach et al. [20] introduced a DC-based trading strategy named 'DBA'. DBA initiates a trade when the magnitude of price change, during the OS event, reaches a particular threshold. DBA closes the position at the DC confirmation point of the next DC event. They applied DBA to 3 currency pairs. Experimental results showed that DBA earns enough 
return to compensate for the risk it took over the trading period. The results also showed that DBA can generate positive returns of up to $14 \%$, within 7 months, after deducting the bid-ask spread.

In 2017, Kampouridis and Otero [21] proposed a DC-based trading strategy named 'DC+GA'. DC+GA runs multiple DC summaries concurrently (using multiple thresholds). For each DC summary, DC+GA keeps tracking the identification of corresponding DC or OS events. For each DC summary, DC+GA uses these DC and OS events jointly with some trading parameters (these parameters are reported in Table 1, page 151, [21]) to make a recommendation to buy or to sell. In other words, each DC summary is used, along with some trading parameters, to produce a buy, or sell, recommendation. DC+GA employs a Genetic Algorithm (GA) module to optimize the selection of thresholds and the values of the trading parameters of each threshold. The objective of this optimization is to maximize the profits produced by $\mathrm{DC}+\mathrm{GA}$.

To evaluate the performance of DC+GA, the authors use five currency pairs. The authors admit that the proposed trading model "...returns a similar average returns with BH." With 'BH' denoting the buy and hold strategy.

In 2017, Golub at al. [22] presented a DC-based trading strategy called 'Alpha Engine'. The Alpha Engine is a counter-trend trading strategy. It opens a position counter the market's trend during the overshoot event. It increases, or decrease, the size of positions during the evolution of prices movements. To decide the size of an order, the Alpha engine uses a sophisticated mechanism which relies on a probability indicator that, in turn, aims to identifying periods of market activity that deviate from normal behavior.

The authors show that the Alpha Engine is profitable over a period of eight years. However, they also admitted that "the model ... yielding an average yearly profit of $10.05 \%$ for the last four years. This is still far from realizing the coastline's potential". Here, the 'coastline' denotes the estimated maximum profits that can be produced under the DC context. In other words, the authors suggested that most of the potentials of the DC framework as basis of trading strategies is not exploited yet [22].

\section{FORECASTING DIRECTIONAL CHANGE: A BRIEF OVERVIEW}

Our objective in this paper is to build a DC-based trading strategy based on the forecasting model presented in Bakhach et al., [11]. This section is essentially a brief summary of this forecasting model. The objective of that forecasting model was to predict whether the current DC trend will continue for a specific percentage before the trend reverses. To formalize this objective, the authors tracked price changes with two thresholds simultaneously: BTheta and STheta (where BTheta > STheta; as in Fig. 5 below).

The authors in [11] defined a Boolean variable named BBTheta. BBTheta is True if, and only if, the current trend, of threshold STheta, continues so that the magnitude of total price change of this trend reaches BTheta before it reverses. Their objective was to predict BBTheta at the DC confirmation point (DCC point) of a DC event of threshold STheta. For example, in Fig. 5, the first DC event observed under the threshold of $0.1 \%$ is $\left[\mathrm{AA}^{0.1}\right]$. Point $\mathrm{A}^{0.1}$ is the DCC point of the DC event $\left[\mathrm{AA}^{0.1}\right]$. The objective is to predict at $\mathrm{A}^{0.1}$ whether the trend of the DC event $\left[\mathrm{AA}^{0.1}\right]$ will continue so that its total magnitude will be at least equal to BTheta. Let BBTheta denote the answer of this question. At $\mathrm{A}^{0.1}$ we don't yet know whether BBTheta ${ }^{l}$ is True. In this case, at point $\mathrm{A}^{0.2}$ we are able to confirm that BBTheta ${ }^{1}$ is True; but not before that. In this example, the objective is to forecast $B B$ Theta ${ }^{l}$ at $\mathrm{A}^{0.1}$. 


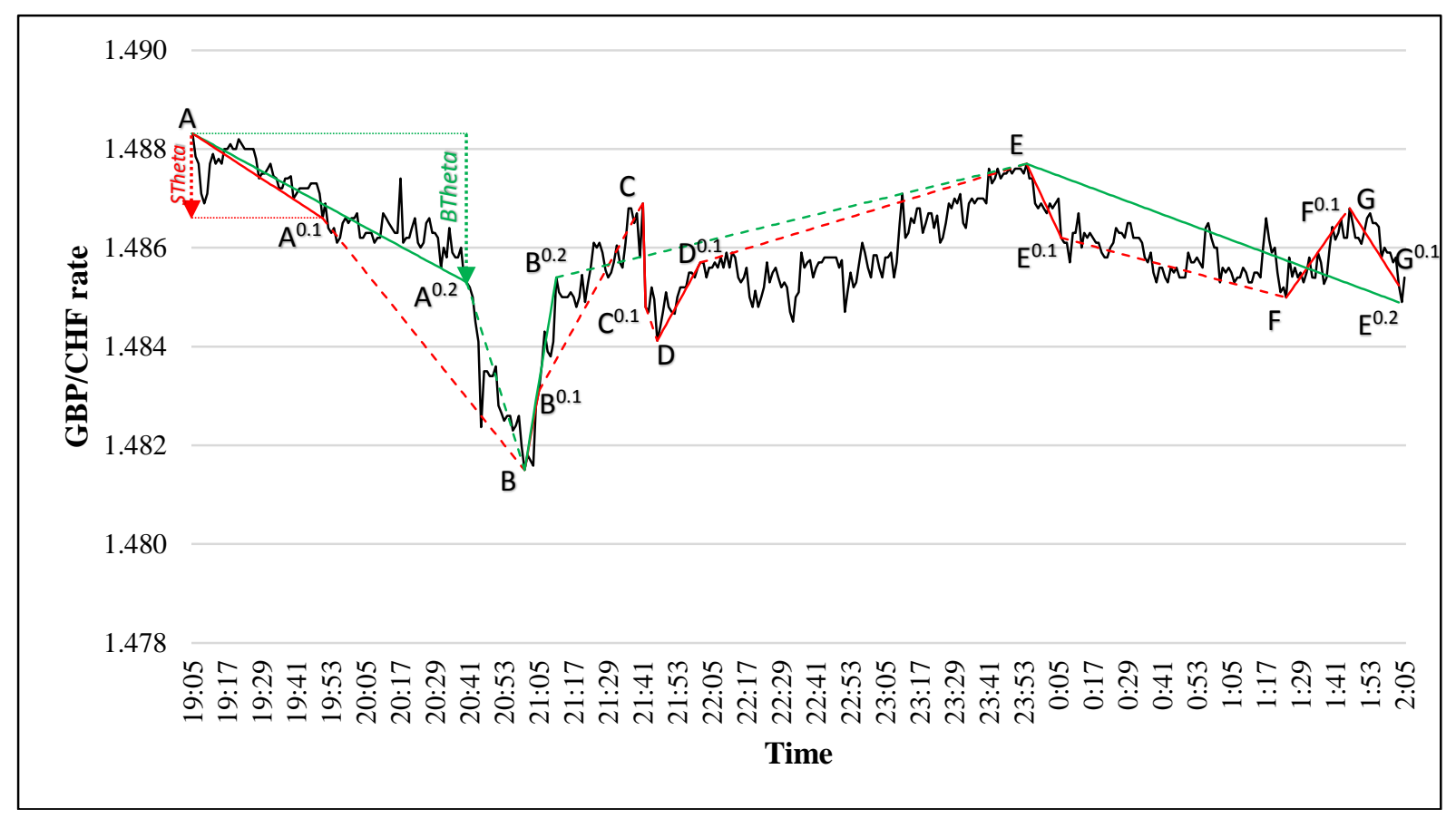

Fig. 5. The synchronization of two DC summaries with two thresholds: STheta $=0.1 \%$ (in red lines) and BTheta $=0.2 \%$ (in green lines) for GBP/CHF rate sampled minute by minute from 1/1/2013 19:05 to 1/2/2013 02:05. Source Bakhach et al., [11].

Table 2: Example of DC events of threshold STheta and computation of corresponding BBTheta ${ }^{i}$ based on Fig. 5. The symbol '--' in column 'DCC point (BTheta)' denotes the fact that the magnitude of price's change of the indexed DC trend, of threshold STheta, does not reach BTheta.

\begin{tabular}{|c|c|c|c|c|}
\hline $\begin{array}{c}\text { DC event index } \\
(\text { STheta })\end{array}$ & $\begin{array}{c}\text { Extreme } \\
\text { point }\end{array}$ & $\begin{array}{c}\text { DCC point } \\
(\text { STheta })\end{array}$ & $\begin{array}{c}\text { DCC point } \\
(\text { BTheta })\end{array}$ & BBTheta \\
\hline 1 & $\mathrm{~A}$ & $\mathrm{~A}^{0.1}$ & $\mathrm{~A}^{0.2}$ & BBTheta $^{1}=$ True \\
\hline 2 & $\mathrm{~B}$ & $\mathrm{~B}^{0.1}$ & $\mathrm{~B}^{0.2}$ & BBThet $^{2}=$ True \\
\hline 3 & $\mathrm{C}$ & $\mathrm{C}^{0.1}$ & -- & BBThet $^{3}=$ False \\
\hline 4 & $\mathrm{D}$ & $\mathrm{D}^{0.1}$ & -- & BBThet $^{4}=$ False \\
\hline 5 & $\mathrm{E}$ & $\mathrm{E}^{0.1}$ & $\mathrm{E}^{0.2}$ & BBThet $^{5}=$ True \\
\hline 6 & $\mathrm{~F}$ & $\mathrm{~F}^{0.1}$ & -- & BBTheta $^{6}=$ False \\
\hline 7 & $\mathrm{G}$ & $\mathrm{G}^{0.1}$ & -- & BBTheta $^{7}=$ False \\
\hline
\end{tabular}

Table 2, shown above, list the identified DC and OS events in Fig 5. We use Table 2 to clarify how to determine the value of BBTheta as in [11]. The first column to the left in Table 2 represents the index of the DC event of threshold STheta (i.e. $1^{\text {st }}, 2^{\text {nd }}$, etc.). The column 'Extreme point' specifies the extreme point corresponding to the indexed DC event. The column 'Extreme point' comprises the points resulted from the DC summary of threshold STheta (Fig. 5). The column 'DCC point (STheta)' denotes the corresponding DCC point of the indexed DC event of threshold STheta. The column named 'DCC point (BTheta)' denotes the corresponding DCC point of the indexed DC event of threshold BTheta. However, if the total magnitude of the indexed DC trend, of threshold STheta, 
is less than BTheta then it will not have an associated DCC point in this column (in such case, it's symbolized as '--').

For example, consider the DC trend of threshold STheta which starts at point C. The DC event $\left[\mathrm{CC}^{0.1}\right]$ is the third indexed DC event (index ' 3 ' in column 'DC event index (STheta)'). In Table 2, points $\mathrm{C}$ and $\mathrm{C}^{0.1}$ denote, respectively, the extreme point and the $\mathrm{DCC}$ point of $\left[\mathrm{CC}^{0.1}\right]$. The $\mathrm{DC}$ trend, which starts at point $\mathrm{C}$, reverses before its total magnitude reaches BTheta. Therefore, the DC event $\left[\mathrm{CC}^{0.1}\right]$ has no associated DCC point of threshold BTheta. Thus, the associated DCC point in the column 'DCC point (BTheta)' is marked as '--'. In this case, the value of BBTheta', reported in the column 'BBTheta', is False. The column 'BBTheta' comprises the set of all instances BBTheta ${ }^{i}$.

Bakhach et al. [11] provided an approach to forecasting the value of BBTheta associated to each DC event of threshold STheta. Forecasting the value of BBTheta ${ }^{i}$ is equivalent to predicting whether the $i^{\text {th }}$ trend, of the DC summary of threshold STheta, will continue so that its total scale will reach BTheta before the trend reverses. For this purpose the authors introduced a novel DC-based indicator as the independent variable. In many cases, the accuracy of the proposed forecasting model was over $80 \%$ (see Table III in [11]). However, in this paper we will not review the detail of their solution as it is not related to the clarification of our proposed trading strategy TSFDC.

\section{INTRODUCING THE TRADING STRATEGY 'TSFDC'}

In this section we introduce a DC based trading strategy named 'Trading Strategy based on Forecasting DC' (TSFDC for short). TSFDC is designed as a contrarian trading strategy (i.e. TSFDC generates buy and sell signals against the market's trend) and is based on the forecasting model established by Bakhach et al. [11]. We present two versions of TSFDC: TSFDC-down and TSFDCup. The former is to be applied if the market exhibits a downward trend under the DC context, with the latter employed in the opposite case. The following explains how TSFDC-down and TSFDC-up operate.

\subsection{TSFDC-down}

TSFDC-down is only applicable when the market is in a downtrend. TSFDC-down relies on the forecasting approach presented in Bakhach et al. [11] to decide when to trigger a buy signal. Let BBTheta ${ }^{i}$ be the value of BBTheta associated with the $i^{\text {th }}$ DC event of threshold STheta (e.g. as in column 'BBTheta', Table 2). Let FBBTheta' denote the forecasted value of BBTheta'. The value of FBBThet ${ }^{i}$ is determined based on the forecasting model presented in [11]. Note that we compute the value of FBBTheta ${ }^{i}$ at the DCC point of the $i^{\text {th }}$ DC event of threshold STheta (e.g. FBBTheta ${ }^{1}$ is calculated at point $\mathrm{A}^{0.1}$ in Fig. 5 above). If FBBTheta ${ }^{i}$ is True, then we expect that the total price change of the $i^{\text {th }}$ DC trend, observed under the threshold STheta, will be at least equal to BTheta. TSFDC-down relies on FBBTheta ${ }^{i}$ to decide when to trigger a buy signal. More particularly, there are two conditions under which TSFDC-down generates buy signal (depending on whether FBBTheta ${ }^{i}$ is True or False):

At the DCC point for the $i^{\text {th }}$ DC trend (STheta), we predict FBBTheta ${ }^{i}$ :

- Rule TSFDC-down.1 (generate buy signal):

If FBBTheta $^{i}=$ False then generate buy signal.

- Rule TSFDC-down.2 (generate buy signal):

If $\left(\right.$ FBBTheta ${ }^{i}=$ True $)$ and (we confirm a new DC event of threshold BTheta) then generate buy signal.

- Rule TSFDC-down.3 (generate sell signal): 
If $\left(P_{c} \geq P_{D C C \uparrow_{*}}\right)$ then generate sell signal.

Where $P_{c}$ indicates the current price and $P_{D C C} \uparrow *$ denotes the lowest prices required to confirm the succeeding uptrend DC event of threshold STheta. If the condition of Rule TSFDC-down.1 is satisfied, then TSFDC-down generates a buy signal at the DCC point observed under threshold STheta. On the other hand, if both conditions of Rule TSFDC-down.2 are fulfilled then TSFDC-down generate buy signal at the DCC point recognized under threshold BTheta. Rule TSFDC-down.3 denotes the case under which we confirm the DCC point for a new DC uptrend of threshold STheta. Rule TSFDC-down.3 is applicable only if a buy signal has been triggered (either by TSFDC-down.1 or TSFDC-down.2). TSFDC-down.3 plays two simultaneous roles: take-profit and stop-loss. When TSFC-down.3 triggers a sell signal, it may incur losses (hence, functioning as stop-loss) or generates profits (thus, working as take-profit).

We use Table 3, shown below, to provide two trading scenarios that demonstrate the function of TSFDC-down's trading rules. Scenario 1: Consider the downtrend DC event [AA $\left.{ }^{0.1}\right]$ (of threshold STheta $=0.1 \%)$.

a) At time 19:50:00 (shown in column 'Time', Table 3), at point $\mathrm{A}^{0.1}$, assume that we predict ${ }^{\mathrm{a}}$ FBBTheta' is True (as shown in column 'FBBTheta').

b) At time 20:40:00, we confirm the DCC point of the DC event, of threshold 0.2\%, [AA ${ }^{0.2}$; which is point $\mathrm{A}^{0.2}$.

c) Based on a) and b), the conditions of Rule TSFDC-down.2 are fulfilled at point $\mathrm{A}^{0.2}$. Thus, TSFDC-down initiates a buy signal at point $\mathrm{A}^{0.2}$.

d) At time 21:05:00, we confirm the DCC point of the next uptrend DC event $\left[\mathrm{BB}^{0.1}\right]$ of threshold $0.1 \%$; which is $\mathrm{B}^{0.1}$ in this case. Following Rule TSFDC-down.3, TSFDC-down will trigger a sell signal at point $\mathrm{B}^{0.1}$.

Scenario 2: Consider the downtrend DC event $\left[\mathrm{CC}^{0.1}\right]$ (of threshold STheta $=0.1 \%$ ).

a) At time 21:42:00 (shown in column 'Time'), at point $\mathrm{C}^{0.1}$, assume that we predict FBBTheta ${ }^{3}$ is False (as shown in column 'FBBTheta').

b) Based on a), the condition of Rule TSFDC-down.1 holds at point $\mathrm{C}^{0.1}$. Thus, TSFDC-down initiates a buy signal at point $\mathrm{C}^{0.1}$.

c) At time 22:01:00, we confirm the DCC point of the next uptrend DC event [DD $\left.{ }^{0.1}\right]$ of threshold $0.1 \%$; which is $\mathrm{D}^{0.1}$ in this case. Following Rule TSFDC-down.3, TSFDC-down will trigger a sell signal at point $\mathrm{D}^{0.1}$.

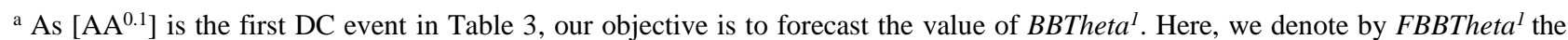
forecasted value of $B B$ Theta ${ }^{1}$.
} 
Table 3: The synchronization of two DC summaries of GBP/CHF mid-prices between 19:05:00 1/1/2013 and 00:06:00 2/1/2013. The two thresholds are: SThet $=0.1 \%$ and BTheta $=0.2 \%$. Unnecessary minutes and prices are omitted. The values in column 'FBBTheta' are hypothetical (for explanation purpose only).

\begin{tabular}{|c|c|c|c|c|c|}
\hline Time & $\begin{array}{l}\text { Mid- } \\
\text { price }\end{array}$ & $\begin{array}{c}\text { DC Summary } \\
(\text { STheta }=0.1 \%)\end{array}$ & $\begin{array}{c}\text { DC Summary } \\
(\text { BTheta }=0.2 \%)\end{array}$ & Point & FBBTheta \\
\hline 19:05:00 & 1.48831 & $\begin{array}{c}\text { start DC event } \\
\text { (DOWNTREND) }\end{array}$ & $\begin{array}{c}\text { start DC event } \\
\text { (DOWNTREND) }\end{array}$ & A & \\
\hline \multicolumn{6}{|c|}{........... } \\
\hline 19:50:00 & 1.48660 & $\begin{array}{c}\text { start OS event } \\
\text { (DOWNTREND) }\end{array}$ & & $\mathrm{A}^{0.1}$ & True \\
\hline \multicolumn{6}{|c|}{$\ldots \ldots \ldots \ldots$} \\
\hline $20: 40: 00$ & 1.48530 & & $\begin{array}{c}\text { start OS event } \\
\text { (DOWNTREND) }\end{array}$ & $\mathrm{A}^{0.2}$ & \\
\hline \multicolumn{6}{|c|}{ n........... } \\
\hline 21:00:00 & 1.48150 & $\begin{array}{c}\text { start DC event } \\
\text { (UPTREND) }\end{array}$ & $\begin{array}{c}\text { start DC event } \\
\text { (UPTREND) }\end{array}$ & B & \\
\hline \multicolumn{6}{|c|}{........... } \\
\hline 21:05:00 & 1.48310 & $\begin{array}{l}\text { start OS event } \\
\text { (UPTREND) }\end{array}$ & & $\mathrm{B}^{0.1}$ & True \\
\hline \multicolumn{6}{|c|}{............ } \\
\hline 21:10:00 & 1.48541 & & $\begin{array}{l}\text { start OS event } \\
\text { (UPTREND) }\end{array}$ & $\mathrm{B}^{0.2}$ & \\
\hline \multicolumn{6}{|c|}{ n........... } \\
\hline 21:41:00 & 1.48690 & $\begin{array}{c}\text { start DC event } \\
\text { (DOWNTREND) }\end{array}$ & & $\mathrm{C}$ & \\
\hline 21:42:00 & 1.48480 & $\begin{array}{c}\text { start OS event } \\
\text { (DOWNTREND) }\end{array}$ & & $\mathrm{C}^{0.1}$ & False \\
\hline \multicolumn{6}{|c|}{............ } \\
\hline 21:46:00 & 1.48412 & $\begin{array}{c}\text { start DC event } \\
\text { (UPTREND) }\end{array}$ & & $\mathrm{D}$ & \\
\hline \multicolumn{6}{|c|}{........... } \\
\hline 22:01:00 & 1.48570 & $\begin{array}{c}\text { start OS event } \\
\text { (UPTREND) }\end{array}$ & & $\mathrm{D}^{0.1}$ & False \\
\hline \multicolumn{6}{|c|}{.............. } \\
\hline $23: 45: 00$ & 1.48770 & $\begin{array}{c}\text { start DC event } \\
\text { (DOWNTREND) }\end{array}$ & & E & \\
\hline \multicolumn{6}{|c|}{............ } \\
\hline 00:06:00 & 1.48620 & $\begin{array}{c}\text { start OS event } \\
\text { (DOWNTREND) }\end{array}$ & & $\mathrm{E}^{0.1}$ & True \\
\hline
\end{tabular}

\subsection{TSFDC-up}

TSFDC-up is the mirror of TSFDC-down in that it is only applicable when the market exhibits an upward trend. TSFDC-up uses FBBTheta ${ }^{i}$ to decide when to generate sell signal. There are two conditions under which TSFDC-up generates a sell signal and one condition in which it will generate buy signal. TSFDC-up operates as follow:

At the DCC point for the $i^{\text {th }}$ DC trend (STheta), we predict FBBTheta ${ }^{i}$ : 
- Rule TSFDC-up.1 (generate sell signal):

If FBBTheta $^{i}=$ False then generate sell signal.

- $\quad$ Rule TSFDC-up.2 (generate sell signal):

If $\left(F B B T h e t a^{i}=\right.$ True $)$ and (we confirm a new DCC point of DC event of threshold $B$ Theta) then generate sell signal.

- Rule TSFDC-up.3 (generate buy signal):

If $\left(P_{c} \leq P_{D C C \downarrow *}\right)$ then generate buy signal.

Note that if the condition of Rule TSFDC-up.1 is True then TSFDC-up generates a sell signal at the DCC point observed under threshold STheta. On the other hand, if the conditions of Rule TSFDCup. 2 are True then TSFDC-up triggers a sell signal at the DCC point observed under threshold BTheta. Rule TSFDC-up.3 denotes the case under which we confirm the DCC point for a new DC downtrend of threshold STheta. Rule TSFDC-up.3 is applicable only if a sell signal has been triggered (either by TSFDC-up.1 or TSFDC-up.2). When TSFDC-up generates buy signal, it may produce profits or losses. Rule TSFDC-up.3 has the same two roles as Rule TSFDC-down.3.

We use Table 3, shown above, to provide two trading scenarios in demonstration of how TSFDCup's rules are applied. Scenario 1: Consider the uptrend DC event $\left[\mathrm{BB}^{0.1}\right]$ (of threshold STheta = $0.1 \%)$ :

a) At time 21:05:00 (shown in column 'Time', Table 3), at point $\mathrm{B}^{0.1}$, assume that we predict FBBTheta $^{2}$ is True $^{\mathrm{b}}$ (as shown in column 'FBBTheta').

b) At time 21:10:00, we confirm the DCC point of the DC event, of threshold $0.2 \%,\left[\mathrm{BB}^{0.2}\right]$; which is point $\mathrm{B}^{0.2}$.

c) Based on a) and b), the conditions of Rule TSFDC-up.2 are fulfilled at point $\mathrm{B}^{0.2}$. Thus, TSFDC-up initiates a sell signal at point $\mathrm{B}^{0.2}$.

d) At time 21:42:00, we confirm the DCC point of the next downtrend DC event $\left[\mathrm{CC}^{0.1}\right]$ of threshold $0.1 \%$; which is $\mathrm{C}^{0.1}$ in this case. Following Rule TSFDC-up.3, TSFDC-up will trigger a buy signal at point $\mathrm{C}^{0.1}$.

Scenario 2: Consider the uptrend DC event $\left[\mathrm{DD}^{0.1}\right]$ (of threshold STheta $=0.1 \%$ ).

a) At time 22:01:00, at point $\mathrm{D}^{0.1}$, assume that we predict FBBTheta ${ }^{4}$ is False (as shown in column 'FBBTheta').

b) Based on a), the condition of Rule TSFDC-up.1 holds at point $\mathrm{D}^{0.1}$. Thus, TSFDC-up initiates a sell signal at point $\mathrm{D}^{0.1}$.

c) At time 00:06:00, we confirm the DCC point of the next downtrend DC event $\left[\mathrm{EE}^{0.1}\right]$ of threshold $0.1 \%$; which is $\mathrm{E}^{0.1}$ in this case. Following Rule TSFDC-up.3, TSFDC-up will trigger a buy signal at point $\mathrm{E}^{0.1}$.

For the best of our knowledge, TSFDC is the first DC-based trading strategy which is founded on a well-formulated forecasting model (the one established by Bakhach et al. [11]). None of the DCbased trading strategies previously reviewed in Section 2.2 (e.g. [20] [21] [22]) employs any forecasting model.

\footnotetext{
${ }^{\mathrm{b}}$ As $\left[\mathrm{BB}^{0.1}\right]$ is the second DC event in Table 3, our objective is to forecast the value of $B B T h e t a^{2}$. Here, we denote by FBBTheta ${ }^{2}$ the forecasted value of BBTheta ${ }^{2}$.
} 


\section{PREPARATION OF THE DATASETS AND OTHER CONSIDERATIONS}

This section provides essential notes regarding the selection and preparation of the datasets that will be used in our experiments. When designing our experiment approach, we paid attention to some important concerns put forward by some studies (e.g. [23] [24]) that highlight serious experimental flaws presented in several published papers. In the context of our experiments, we consider the following points:

\subsection{Data selection}

Pardo [23] emphasizes the importance of evaluating the performance of a trading strategy using a set of assets with different trends. Such variation in the selected dataset will help to test the performance of the trading strategy under different market scenarios. This variation helps in avoiding any bias towards particular patterns. Therefore, we consider eight currency pairs, namely: EUR/CHF, GBP/CHF, EUR/USD, GBP/AUD, GBP/JPY, NZD/JPY, AUD/JPY, and EUR/NZD. These currency pairs are sampled minute-by-minute during a period of 31 months between 01/01/2013 and $31 / 07 / 2015$. These selected currency rates exhibit various trends during the trading period that lasts from $1 / 1 / 2015$ to $31 / 7 / 2015$. Our focus, in this section, is to examine the variation of the trends of these currency pairs during the trading period. The training period took place between $1 / 1 / 2013$ and $31 / 12 / 2014$. We do not examine the trends of these currency pairs during the training period as it is not very related to the objective of evaluating the performance of TSFDC. Holidays and weekends are not included in our datasets.

In this section, we investigate the variation of the trends of the selected currency pairs. Variation is important because some studies (e.g. [23]) have shown that trend changes can have a large and often negative impact on trading performance. Table 4, shown below, provides the descriptive statistics of the 1-minute returns of these currency pairs. The column 'Mean $\times 10^{-5}$ ' denote the mean (in \%) of one-minute-based returns. By examining the values shown in this column, we see that our set contains a mix of overall negative trends (EUR/CHF, EUR/USD, NZD/JPY, and AUD/JPY) and positive trends (GBP/CHF, GBP/AUD, GBP/JPY, and EUR/NZD) over the selected trading period of seven months (from 1/1/2015 to 31/7/2015). The values of the skewness and kurtosis ${ }^{\mathrm{c}}$ in Table 4 suggest that the one-minute returns of these currency pairs have different densities' distributions, which reflect the variation of the fluctuations of these currency pairs.

Table 4: Descriptive statistics of 1-minute returns for the currency pairs utilized in the experiments (measured during the trading period of seven months). The '1-minute returns' is calculated as the prices' change, in percentage, between each two consecutive minutes in which at least one transaction is recorded. In other words, if no trade has been registered for one minute, it would not be counted in the calculus of returns. These numbers are computed based on our minute-by-minute datasets provided by www.kibot.com.

\begin{tabular}{|c|c|c|c|c|}
\hline Currency pairs & Mean $\times \mathbf{1 0}^{-\mathbf{5}}$ & Std. Dev. $\times \mathbf{1 0}^{-\mathbf{3}}$ & Skewness & Kurtosis \\
\hline EUR/CHF & -3.10 & 0.695096 & 27.248 & 23359.630 \\
\hline GBP/CHF & 0.20 & 0.534922 & -59.302 & 16372.470 \\
\hline EUR/USD & -4.17 & 0.239995 & -0.883 & 151.965 \\
\hline GBP/AUD & 5.01 & 0.262100 & 0.650 & 129.999 \\
\hline GBP/JPY & 1.84 & 0.197687 & -1.265 & 156.070 \\
\hline NZD/JPY & -5.30 & 0.303782 & -1.532 & 171.195 \\
\hline AUD/JPY & -2.56 & 0.267428 & -0.721 & 91.941 \\
\hline EUR/NZD & 4.55 & 0.356778 & 0.913 & 90.501 \\
\hline
\end{tabular}

\footnotetext{
${ }^{\mathrm{c}}$ For more information about skewness and kurtosis see: http://www.math.uah.edu/stat/expect/Skew.html
} 
In addition, Fig. 6 shows the normalized daily rates of the selected eight currency pairs throughout the considered trading period. It provides a general indication as to the existence of a variety of trends in our dataset over the considered trading period. The fluctuations of these trends, as shown in Fig. 6 , ensures that we avoid possible bias in our experiment, which would have occurred had we only picked currency pairs with similar trends during the selected trading period.

\section{Normalized daily exchange rates}

1.2

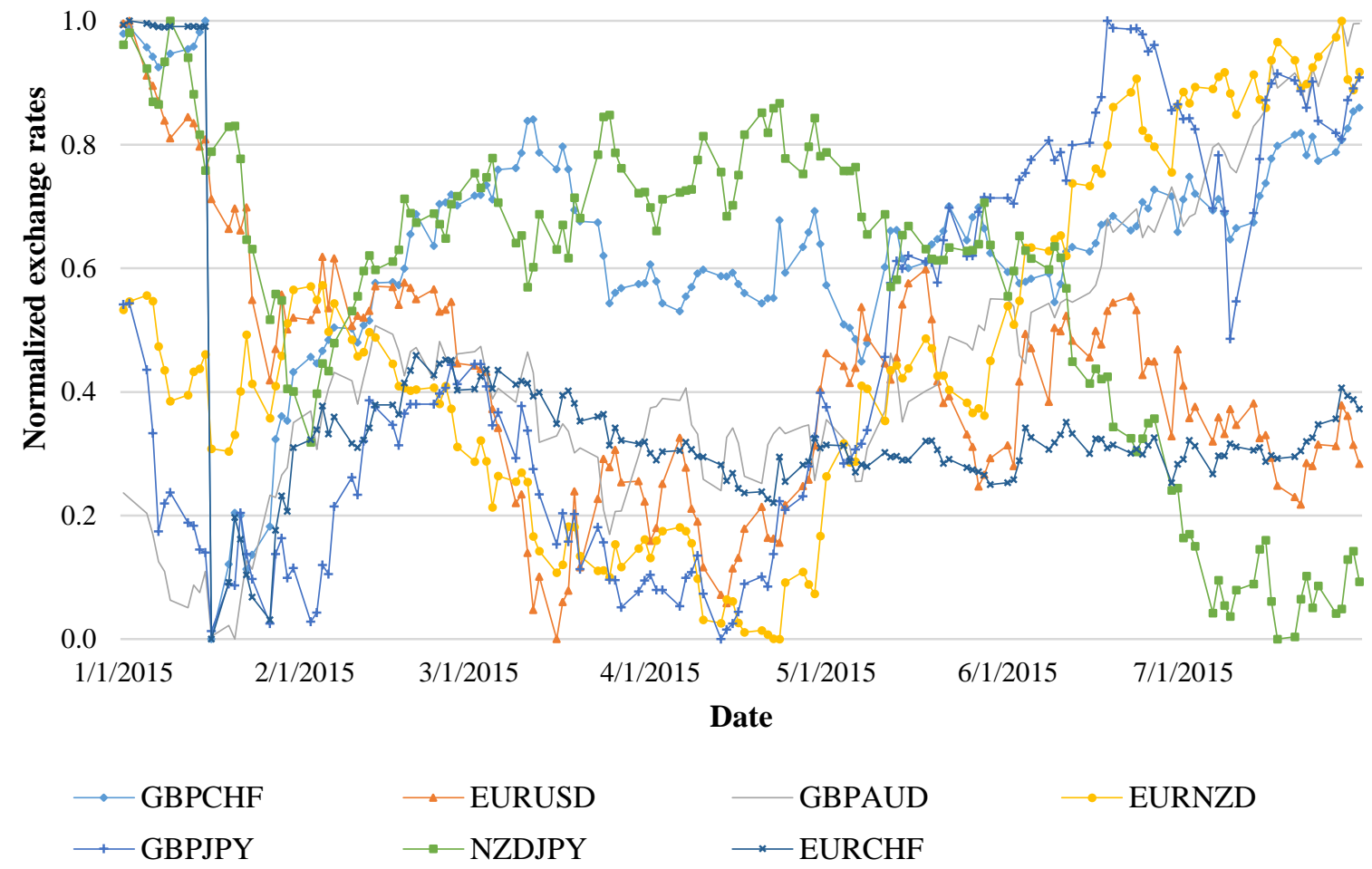

Fig. 6. Normalized daily rate changes of the 8 selected currency pairs between 1/1/2015 and 31/7/2015. This figures aims to illustrate the fluctuations of trends of selected currency pairs. In order to avoid excessive points, we use a daily exchange rate instead of minutebased exchange rates.

\subsection{Measuring the performance of a trading strategy}

Many studies define success solely on the grounds of forecast accuracy and win ratios, which, practically, has little value ( [25] [26]). In fact, an investor might be interested in other metrics that evaluate the risk and risk-adjusted performance of a given trading strategy ( [27] [28]). In this paper, we evaluate the performance of TSFDC using a range of evaluation metrics marked as adequate for a decent evaluation of the performance of a given trading model ( [23] [27]).

- Rate of returns: The rate of returns $(R R)$ symbolizes the bottom line for a trading system over a definite period of time. Let Total Profit $(T P)$ represents the profitability of total trades. $T P$ is computed by removing the gross loss of all losing trades from the gross profit of all winning trades (3). $T P$ can be negative when the loss is greater than the gain. We denote by $R R$ (4) the gain or loss on an investment over a given evaluation period expressed as a percentage of the amount invested. In (4) $I N V$ denotes the initial capital employed in investment. 


$$
\begin{gathered}
T P=\text { sum of all profits }- \text { sum of all losses } \\
R R=\frac{T P}{I N V} * 100
\end{gathered}
$$

- Profit factor: The profit factor (5) is defined as the gross profit divided by the gross loss for the entire trading period. This metric measures the amount of profit per unit of risk, with values greater than one signifying a profitable system.

$$
\text { Profit factor }=\frac{\text { sum of all profits }}{\text { sum of all losses }}
$$

- Max drawdown (\%): The drawdown (6) is defined as the difference, in percentage, between the highest profit, previous to the current time point, and the current profit value. The Maximum Drawdown $(M D D)$ is the largest drawdown observed during a specific trading period. $M D D$ measures the risk as the 'worst case scenario' for a trading period. This metric can help measure the amount of risk incurred by a system and determine if a system is practical. If the largest amount of money that a trader is willing to risk is less than the maximum drawdown, the trading system is not suitable for the trader. In (6) and (7), the subscript $i$ denotes the trade-index. For the $i^{\text {th }}$ executed trade (i), Current capital $i$ denote the amount of capital counted after the execution of that trade. The maximum capital refers to the peak capital's value that has been reached since the beginning of trading up to the $i^{\text {th }}$ trade. Thus, drawdown $n_{i}(6)$, is interpreted as the peak-to-trough decline during a specific recorded period of an investment. Note that, based on (6), we have drawdown $i \leq 0$ for all $i$. The MDD (7) is the minimum value among all computed drawdown $n_{i}$. The nbTrades, in (7), denotes the number of executed trades by a trading strategy.

$$
\begin{gathered}
\text { drawdown }_{i}=\frac{\text { current capital }_{i}-\text { maximum capital }}{\text { maximum capital }} \\
M D D=\operatorname{Min}\left(\text { drawdown }_{i}\right) \quad \forall i=1,2, \ldots, \text { nbTrades }
\end{gathered}
$$

- Win ratio: The 'Win ratio' is calculated by dividing the number of winning trades by the total number of trades for a specified trading period. It represents the probability of having a profitable trade.

$$
\text { Win ratio }=\frac{\text { number of wining tardes }}{\text { total number of all trades }}
$$

- Sharpe ratio [29]: The Sharpe ratio (9) is a measure for calculating risk-adjusted return. The basic purpose of the Sharpe ratio is to allow an investor to analyse how much greater a return he or she is obtaining in relation to the level of additional risk taken to generate that return. The Sharpe ratio can be seen as the average return earned in excess of the risk-free rate per unit of volatility or total risk. To date, it remains one of the most popular risk-adjusted performance measures due to its practical use. Some studies (e.g. [30] [31]) show that, despite its shortcomings, the Sharpe ratio indicates similar performance rankings to the more sophisticated performance risk-adjusted ratios (e.g. Treynor ratio [32]).

$$
\text { Sharpe ratio }=\frac{R_{p}-R_{f}}{\sigma_{p}}
$$


Where: $R_{p}$ denotes the expected portfolio retunes; $R_{f}$ is the risk-free rate; $\sigma_{p}$ designs the standard deviation of the portfolio's returns. One intuition of this calculation is that a portfolio engaging in "zero risk" investment, such as the purchase of U.S. Treasury bills (for which the expected return is the risk-free rate), has a Sharpe ratio of exactly zero. Generally, the greater the value of the Sharpe ratio, the more attractive the risk-adjusted return.

- Sortino ratio [33]: The downside risk (10) is defined as the standard deviation of negative asset returns. The Sortino ratio (11) uses the downside risk to measure the risk associated to a given investment. In (11), the 'return' represents the profits generated by a given trading strategy and the 'target return' is the minimum acceptable return (MAR).

$$
\begin{gathered}
\text { Downside risk }=\sqrt{\frac{\sum_{i=1}^{m}\left(\text { return }_{i}-\text { target return }_{i}\right)^{2} f(t)}{m}} ; \\
\text { Where } f(t)=\left\{\begin{array}{cl}
1 & \text { if return }<\text { target return } \\
0 & \text { if return } \geq \text { target retun }
\end{array}\right. \\
\text { Sortino ratio }=(\text { return }- \text { target return }) \div \text { Downside risk }
\end{gathered}
$$

\subsection{Model training and testing process}

Pardo [23] suggests the adoption of a rolling window approach as being more reliable to test a trading strategy. This approach is usually used for evaluating trading systems and establishes a more rigorous and convincing methodology. This method involves splitting the data into overlapping training-applied sets and, on each cycle, moving each set forward through the time series. This methodology tends to result in more robust models due to more frequent retraining and large out-ofsample data sets (increasing training processing requirements but also resulting in models which adapt more quickly to changing market conditions). Therefore, in our experiments, we train and test the trading model on a monthly rolling window basis as we will explain below.

\subsection{Preparing the rolling windows}

Our experiments examine eight currency pairs: EUR/CHF, GBP/CHF, EUR/USD, GBP/AUD, GBP/JPY, NZD/JPY, AUD/JPY, and EUR/NZD and consider the minute-by-minute transaction prices of these currency pairs for 31 months: from 1/1/2013 to 31/7/2015. Given that the preparation process of the rolling windows for each currency pair is the same, we will explain a two-steps preparation of the rolling windows for the currency pairing GBP/CHF to detail our method.

\section{Step 1: Producing DC summary for the dataset}

We run the Directional Change (DC) summary on the initial dataset of GBP/CHF. Section 2.1 provides a detailed description of the DC summary. In simple terms, given a threshold STheta, we achieve, through DC summary, the identification of all DC and OS events in the initial dataset. Arbitrarily, we set STheta $=0.10 \%$ and produce the DC summary to the initial dataset of GBP/CHF. Let $G B P C H F \_D C 0.1$ be the output of this DC summary. Part of GBPCHF_DCO.1 is illustrated in Table 5. GBPCHF_DCO.1 comprises the date, time and the price of each observation of the initial dataset. In Table 5, the column 'Event Type' marks the observation of DC and OS events that starts at the specified date and time (see Section 2.1 for more info about DC summary). 
Table 5: An example of DC summary using GBP/CHF mid-prices sampled minute-by-minute from 21:41:00 to 22:01:00 (UK time).

\begin{tabular}{|c|c|c|c|}
\hline Date & Time & Mid-price & Event Type \\
\hline $1 / 1 / 2013$ & $21: 41: 00$ & 1.48690 & start DC event (DOWNTREND) \\
\hline $1 / 1 / 2013$ & $21: 42: 00$ & 1.48480 & start OS event (DOWNTREND) \\
\hline $1 / 1 / 2013$ & $21: 43: 00$ & 1.48470 & \\
\hline $1 / 1 / 2013$ & $21: 44: 00$ & 1.48520 & \\
\hline $1 / 1 / 2013$ & $21: 45: 00$ & 1.48495 & \\
\hline $1 / 1 / 2013$ & $21: 46: 00$ & 1.48412 & start DC event (UPTREND) \\
\hline $1 / 1 / 2013$ & $21: 47: 00$ & 1.48440 & \\
\hline $1 / 1 / 2013$ & $21: 48: 00$ & 1.48470 & \\
\hline $1 / 1 / 2013$ & $21: 49: 00$ & 1.48510 & \\
\hline $1 / 1 / 2013$ & $21: 50: 00$ & 1.48480 & \\
\hline $1 / 1 / 2013$ & $21: 51: 00$ & 1.48470 & \\
\hline $1 / 1 / 2013$ & $21: 52: 00$ & 1.48466 & \\
\hline $1 / 1 / 2013$ & $21: 53: 00$ & 1.48500 & \\
\hline $1 / 1 / 2013$ & $21: 54: 00$ & 1.48520 & \\
\hline $1 / 1 / 2013$ & $21: 55: 00$ & 1.48520 & \\
\hline $1 / 1 / 2013$ & $21: 56: 00$ & 1.48520 & \\
\hline $1 / 1 / 2013$ & $21: 57: 00$ & 1.48550 & \\
\hline $1 / 1 / 2013$ & $21: 58: 00$ & 1.48550 & \\
\hline $1 / 1 / 2013$ & $21: 59: 00$ & 1.48540 & \\
\hline $1 / 1 / 2013$ & $22: 00: 00$ & 1.48560 & \\
\hline $1 / 1 / 2013$ & $22: 01: 00$ & 1.48570 & start OS event (UPTREND) \\
\hline
\end{tabular}

Step 2: Composing the rolling windows

Motivated by the recommendation of Pardo [23], we use a rolling window approach (see Fig. 7 below) to evaluate the performance of our proposed trading strategy. As the dataset GBPCHF_DCO.1 covers 31 months, we compose seven rolling windows - each of which comprises a training window (24 months in length) and an applied window (1 month in length). So that the overall trading period of the seven rolling windows, combined together, is seven months. The lengths of the training and applied windows are set arbitrarily. Note that we measure the length of the training and applied windows as a function of months, not as a fixed number of days. For example, the training period of the second rolling window lasts from 1/2/2013 to 31/1/2015 (i.e. 24 months). The associated applied window lasts from 1/2/2015 00:01:00 to 28/2/2015 23:59:00 (i.e. the month of February 2015). Let $G B P C H F \_R W D C 0.1$ represent the set of these seven rolling windows. Similarly, we construct seven sets of rolling windows (one for each of the remaining currency pairs). For example, let $E U R C H F \_R W D C 0.1$ be the set of the seven rolling windows corresponding to EUR/CHF and let EURUSD_RWDC0.1 be the set of the seven rolling windows corresponding to EUR/USD and so on. These sets are compiled in the same two steps as GBPCHF_RWDC0.1 with a threshold STheta $=$ $0.1 \%$.

Finally, we get the following eight sets of rolling windows: EURCHF_RWDC0.1, GBPCHF_RWDC0.1, EURUSD_RWDC0.1, GBPAUD_RWDC0.1, GBPJPY_RWDC0.1, NZDJPY_RWDC0.1, AUDJPY_RWDC0.1, and EURNZD_RWDC0.1. 


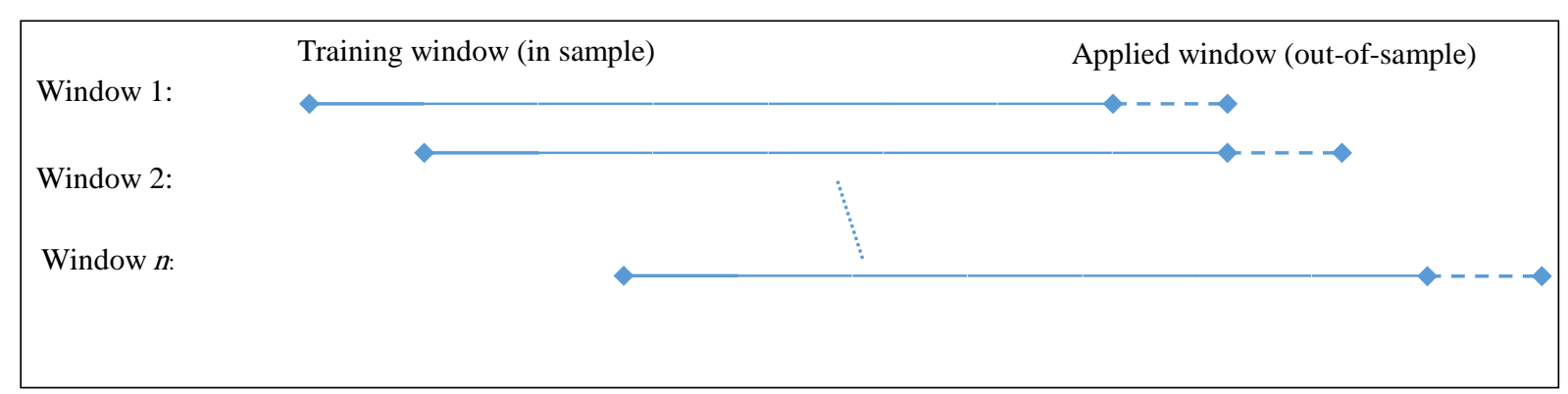

Fig. 7. Illustration of $n$ rolling windows. The dashed lines represent the applied windows.

\section{EVALUATION OF TSFDC: THE EXPERIMENTS}

In this section, we examine the performance of TSFDC. The objective is to evaluate the profitability and risk of both versions of TSFDC (i.e. TSFDC-down and TSFDC-up) using the rolling windows previously composed in Section 5.4. We provide the details of the experiments after describing the adopted money management approach.

\subsection{Money management approach}

We apply the following money management approach to both TSFDC-down and TSFDC-up. When TSFDC-down initiates a buy signal, we convert the entire capital from the counter currency to the base currency. When TSFDC-down generates a sell signal we convert the entire available amount of base currency to counter currency. Likewise in the case of TSFDC-up. Although this sounds like a naïve approach to money management, our main objective is to prove that TSFDC is a successful trading strategy. Future works may address the development of a better money management approach (e.g. [28]).

When we apply any version of TSFDC, we make sure that no position is left open at the end of the trading period. Should we encounter an open position at the end of the trading period, then the last transaction will not be considered when computing the results — instead, we roll back to the previous transaction. In other words, we do not count this last trade when measuring any of the evaluation metrics (previously introduced in Section 5.2). Thus, as a result of this approach, if TSFDC opens a position it will not be able to open any other positions until the current position is closed.

In our experiment, we do not account the transaction cost. Eventually, counting transaction cost will decrease the returns of a trading strategy. However, some studies (e.g. [34] [35] [36]) have shown that counting transaction costs is not expected to have a substantial negative impact on the profitability of technical trading in the FX market. Besides, some market makers (e.g. OANDA) do not charge their customers for transaction costs for FX trading (see https://www.oanda.com/resources/news/pr/fxtrade03292001). We should also highlight that we ignore the effect of 'slippage' in our trading simulations. In trading, slippage refers to the difference between what a trader expects to pay for a trade and the actual price at which the trade is executed. Normally, the slippage happens because there might be a slight time delay between the trader initiating the trade and the time the broker receives the order. During this time delay, the price may have changed. It can either work in favour of, or against, the trader [37].

\subsection{Experiment 1: Evaluation of the performance of TSFDC}

The objective of this experiment is to evaluate the performance of TSFDC-down and TSFDC-up. For this purpose, we apply both versions to the eight currency pairs sampled minute-by-minute: 
EUR/CHF, GBP/CHF, EUR/USD, GBP/AUD, GBP/JPY, NZD/JPY, AUD/JPY, and EUR/NZD. We consider the eight sets of rolling windows: EURCHF_RWDC0.1, GBPCHF_RWDC0.1, EURUSD_RWDC0.1...etc. (previously composed in Section 5.4). For each of these eight sets, the training period of each rolling window (24 months) is used to train the forecasting model of Bakhach et al. [11]. Next, the forecasting model is used to compute the value of FBBTheta (i.e. to forecast BBTheta) for each DC event, of threshold STheta, during the trading period (i.e. the associated applied window of 1 month). TSFDC uses FBBTheta to decide when to initiate a trade, as described in Section 4, during the trading period. The overall trading period of each set is seven months in length: from $1 / 1 / 2015$ to $31 / 7 / 2015$. For each of the eight sets, BTheta is set, arbitrarily, to $0.13 \%$. We measure the evaluation metrics previously listed in Section 5.2 to evaluate the performance of TSFDC. Note that although our initial datasets in this experiment (i.e. the eight currency exchange rates) are sampled as a time series (with an interval of one minute), the TSFDC's trading rules (presented in Section 4) are based on variables (e.g. $\left.P_{D C C} \uparrow^{*}\right)$ which originate from the DC concept.

In this paper, we consider the buy and hold $(\mathrm{B} \& \mathrm{H})$ approach as our benchmark. Thus, we apply $\mathrm{B} \& \mathrm{H}$ to each considered currency pair (buying at the opening price on a monthly basis; holding it over the course of the trading month, and selling at the closing price). For each currency pair, we compute the monthly returns resulting from applying the $\mathrm{B} \& \mathrm{H}$ to the specified trading periods (during the seven months: from January 2015 to July 2015).

\subsection{Experiment 2: Compare the return and risk of both versions of TSFDC}

The objective of this experiment is to test whether there is a significant difference in the performance of TSFDC-down versus TSFDC-up and vice versa. To this end, we compare the return and risk of both versions of TSFDC. In this experiment, we consider the monthly rate of returns $(R R)$ and maximum drawdown $(M D D)$ resulted from applying both versions of TSFDC to the eight currency pairs from the previous experiment. In order to validate our test statistically, we chose to apply the non-parametric Wilcoxon test. More particularly, we apply the Wilcoxon signed rank test [38] (also called the Mann-Whitney $U$ test).

In this experiment, we apply the Wilcoxon test twice. Firstly, we apply the Wilcoxon test with the null hypothesis being that there is no difference between the two sets of monthly $R R$ of TSFDC-down and TSFDC-up. In this instance, we assess the monthly $R R$ generated by applying TSFDC-down to the eight currency rates as the first set. This set consists of 56 observations $(8$ currency rates $\times 7$ monthly $R R$ for each currency rate). Similarly, the second set comprises the monthly $R R$ generated by applying TSFDC-up to the eight currency rates (a total of other 56 observations). We report the details of these two sets in Appendix A.

Secondly, we seek to compare the risk of both versions of TSFDC. Taking the maximum drawdown as the main indicator of risk, we compose a first set by applying TSFDC-down to the eight currency rates. This set comprises 56 observations ( 8 currency rates $\times 7$ monthly $M D D$ for each currency rate). We compose a second set of monthly $M D D$ data by applying TSFDC-up to the eight currency rates and apply the Wilcoxon signed rank test to each, with the null hypothesis that there is no difference between the two sets of monthly MDD of TSFDC-down and TSFDC-up (Appendix A comprises the details of these two sets). 


\section{EVALUATION OF TSFDC: RESULTS AND DISCUSSION}

\subsection{Experiment 1: Evaluation of the profitability and risk of TSFDC}

The objective of our experiments is to evaluate the performance of TSFDC-down and TSFDC-up using eight currency pairs sampled minute-by-minute. To this end, we applied the two versions of TSFDC to the eight sets of rolling windows composed in Section 5.4. We adopt the money management approach outlined in Section 6.1 and measure the evaluation metrics listed in Section 5.2. We did not consider the bid and ask price nor the transaction cost in any of these experiments. In order to avoid tedious details, this section reports TSFDC's general trading performance over the eight currency pairs.

\section{Experiment 1: The results}

For each currency pair, we use the same values of STheta $(0.1 \%)$ and BTheta $(0.13 \%)$. These values are chosen arbitrarily. Bear in mind that, for each currency pair, we compose seven rolling windows. Each window comprises a trading period of one month. At the beginning of the first trading period, i.e. January 2015 , both TSFDC-down and TSFDC-up start with a capital $=1,000,000^{\mathrm{d}}$; this represents the initial, hypothetically, invested amount of money. Table 6 shows the general performance of applying both versions of TSFDC to the eight exchange rates.

Table 6: Trading performance of TSFDC-down and TSFDC-up models following the seven months out-of-sample period of the eight currency pairs.

\begin{tabular}{|c|c|c|c|c|c|c|}
\hline $\begin{array}{c}\text { Currency } \\
\text { Pair }\end{array}$ & $\begin{array}{c}\text { Trading } \\
\text { Strategy }\end{array}$ & $\begin{array}{c}\text { RR } \\
(\%)\end{array}$ & $\begin{array}{c}\text { Profit } \\
\text { Factor }\end{array}$ & $\begin{array}{c}\text { Total Number } \\
\text { of Trades }\end{array}$ & $\begin{array}{c}\text { Max } \\
\text { Drawdown (\%) }\end{array}$ & Win Ratio \\
\hline \multirow{2}{*}{ EUR/CHF } & TSFDC-down & 84.59 & 1.93 & 2056 & -13.4 & 0.73 \\
\cline { 2 - 7 } & TSFDC -up & 63.03 & 1.83 & 2009 & -15.1 & 0.71 \\
\hline \multirow{2}{*}{ GBP/CHF } & TSFDC-down & 94.03 & 1.73 & 2489 & -12.1 & 0.72 \\
\cline { 2 - 7 } & TSFDC -up & 115.19 & 1.69 & 2531 & -10.8 & 0.70 \\
\hline \multirow{3}{*}{ EUR/USD } & TSFDC-down & 24.04 & 1.26 & 1431 & -5.0 & 0.65 \\
\cline { 2 - 7 } & TSFDC -up & 36.09 & 1.32 & 1453 & -5.8 & 0.67 \\
\hline \multirow{3}{*}{ GBP/AUD } & TSFDC-down & 92.63 & 1.86 & 3021 & -3.4 & 0.70 \\
\cline { 2 - 7 } & TSFDC -up & 63.03 & 1.54 & 2960 & -3.5 & 0.68 \\
\hline \multirow{2}{*}{ GBP/JPY } & TSFDC-down & 32.48 & 1.53 & 1585 & -4.8 & 0.69 \\
\cline { 2 - 7 } & TSFDC -up & 28.91 & 1.42 & 1601 & -5.7 & 0.69 \\
\hline \multirow{2}{*}{ NZD/JPY } & TSFDC-down & 183.13 & 2.20 & 3046 & -4.0 & 0.73 \\
\cline { 2 - 7 } & TSFDC -up & 190.73 & 2.08 & 3010 & -4.9 & 0.74 \\
\hline \multirow{2}{*}{ AUD/JPY } & TSFDC-down & 104.11 & 1.70 & 2885 & -5.0 & 0.71 \\
\cline { 2 - 7 } & TSFDC -up & 116.35 & 1.81 & 2860 & -5.2 & 0.72 \\
\hline \multirow{2}{*}{ EUR/NZD } & TSFDC-down & 489.13 & 2.98 & 3961 & -4.6 & 0.77 \\
\cline { 2 - 7 } & TSFDC -up & 571.89 & 2.86 & 4218 & -5.1 & 0.77 \\
\hline
\end{tabular}

${ }^{\mathrm{d}}$ For each currency pairs, in case of trading with TSFDC-down, we assume that we start with 1,000,000 monetary units of the counter currency. For example: in the case of EUR/CHF, we start with 1,000,000 CHF. Similarly, in the case of NZD/JPY, we start with 1,000,000 JPY. However, in the case of TSFDC-up we assume that we start with 1,000,000 monetary units of the base currency. . For example: in the case of EUR/CHF, we start with 1,000,000 EUR. 
Table 6 reports the general performance of both versions of TSFDC. The column 'Currency Pair' denotes the considered currency pair. The column 'Trading Strategy' indicates which version of TSFDC is applied. The column 'RR (\%)' is the total returns expressed as a percentage of the capital employed. The column 'Profit Factor' is calculated by dividing the sum of all generated profits by the sum of incurred losses during the overall trading period of seven months. The column 'Max Drawdown (\%)' refers to the worst scenario measured as the worst peak-to-trough decline in capital during the trading period of seven months. The column 'Win Ratio' is the overall probability of having a wining trade (See Section 3.4 for more info about these evaluation metrics). The last row in Table 6 is interpreted as follows: applying TSFDC-up to EUR/NZD generates a total return of $571.89 \%$ during the seven-month trading period. In this case, TSFDC-up executes 4218 trades with an overall Win Ratio of 0.77 . The maximum drawdown in capital (throughout the seven months) is $-5.1 \%$.

Table 7: Monthly RR (\%) of applying TSFDC-down to the eight currency pairs shown in Table 6.

\begin{tabular}{|l|c|c|c|c|c|c|c|c|}
\hline $\begin{array}{l}\text { Trading } \\
\text { period }\end{array}$ & $\begin{array}{c}\text { EUR/ } \\
\text { CHF }\end{array}$ & $\begin{array}{c}\text { GBP/ } \\
\text { CHF }\end{array}$ & $\begin{array}{c}\text { EUR/ } \\
\text { USD }\end{array}$ & $\begin{array}{c}\text { GBP/ } \\
\text { AUD }\end{array}$ & $\begin{array}{c}\text { GBP/ } \\
\text { JPY }\end{array}$ & $\begin{array}{c}\text { NZD/ } \\
\text { JPY }\end{array}$ & $\begin{array}{c}\text { AUD/ } \\
\text { JPY }\end{array}$ & $\begin{array}{c}\text { EUR/ } \\
\text { NZD }\end{array}$ \\
\hline Jan 2015 & 4.47 & 13.59 & 1.12 & 19.70 & 7.72 & 19.14 & 15.36 & 24.12 \\
\hline Feb 2015 & 14.40 & 19.02 & 7.54 & 10.51 & 6.40 & 26.90 & 16.47 & 50.04 \\
\hline Mar 2015 & 17.59 & 14.96 & -0.36 & 10.14 & 4.04 & 19.95 & 10.51 & 49.76 \\
\hline Apr 2015 & 7.58 & 6.71 & 4.20 & 13.52 & 7.05 & 30.41 & 16.69 & 59.39 \\
\hline May 2015 & 13.37 & 9.85 & 5.73 & 15.97 & 8.38 & 24.27 & 25.51 & 79.92 \\
\hline Jun 2015 & 12.41 & 15.17 & 7.85 & 11.52 & 0.99 & 17.20 & 10.48 & 104.91 \\
\hline Jul 2015 & 14.77 & 14.73 & 0.96 & 11.27 & -2.10 & 45.26 & 9.09 & 120.99 \\
\hline Sum & 84.59 & 94.03 & 27.04 & 92.63 & 32.48 & 183.13 & 104.11 & 489.13 \\
\hline
\end{tabular}

Table 8: Monthly RR (\%) of applying TSFDC-up to the eight currency pairs shown in Table 6.

\begin{tabular}{|l|c|c|c|c|c|c|c|c|}
\hline $\begin{array}{l}\text { Trading } \\
\text { period }\end{array}$ & $\begin{array}{c}\text { EUR/ } \\
\text { CHF }\end{array}$ & $\begin{array}{c}\text { GBP/ } \\
\text { CHF }\end{array}$ & $\begin{array}{c}\text { EUR/ } \\
\text { USD }\end{array}$ & $\begin{array}{c}\text { GBP/ } \\
\text { AUD }\end{array}$ & $\begin{array}{c}\text { GBP/J } \\
\text { PY }\end{array}$ & $\begin{array}{c}\text { NZD/ } \\
\text { JPY }\end{array}$ & $\begin{array}{c}\text { AUD/ } \\
\text { JPY }\end{array}$ & $\begin{array}{c}\text { EUR/ } \\
\text { NZD }\end{array}$ \\
\hline Jan 2015 & 4.26 & 31.54 & 6.81 & 13.34 & 11.39 & 26.96 & 21.48 & 26.27 \\
\hline Feb 2015 & 9.75 & 16.30 & 9.27 & 10.06 & 3.64 & 18.06 & 14.88 & 68.74 \\
\hline Mar 2015 & 16.87 & 21.67 & 1.69 & 9.09 & 6.00 & 24.06 & 17.30 & 64.56 \\
\hline Apr 2015 & 5.71 & 12.34 & 1.66 & 9.23 & 3.07 & 22.98 & 12.25 & 78.72 \\
\hline May 2015 & 7.61 & 7.59 & 9.67 & 9.51 & 4.11 & 24.92 & 21.15 & 82.81 \\
\hline Jun 2015 & 10.15 & 14.13 & 6.13 & 5.97 & 4.16 & 32.66 & 17.32 & 101.88 \\
\hline Jul 2015 & 8.68 & 11.62 & 0.86 & 5.83 & -3.46 & 41.09 & 11.97 & 148.91 \\
\hline Sum & 63.03 & 115.19 & 36.09 & 63.03 & 32.37 & 190.73 & 116.35 & 571.89 \\
\hline
\end{tabular}

The results of monthly Rates of Return $(R R)$ of applying TSFDC-down and TSFDC-up to these currencies are shown in Tables 7 and 8 respectively. These returns will be used to compute the Sharpe and Sortino ratios. The Sharpe and Sortino ratios of both versions of TSFDC are reported in Table 9. The minimum acceptable return (MAR) and the risk-free rate are set to 5\% per annum. In this paper, we adopt the buy and hold approach as a benchmark. For each currency pair, we apply the buy and hold approach on a monthly basis over the considered trading period from 1/1/2015 to 31/7/2015 
(seven months). Table 10, shown below, summarizes the monthly returns of applying the buy-andhold $(\mathrm{B} \& \mathrm{H})$ approach to the eight currency pairs. The column 'Sum', in Table 10, shows the total $R R$ of applying $\mathrm{B} \& \mathrm{H}$ to the specified currency pair.

Table 9: The Sortino and Sharpe ratio of the two versions of TSFDC. The math symbol ' $\infty$ ' denotes positive infinity.

\begin{tabular}{|l|c|c|c|c|}
\hline \multirow{2}{*}{$\begin{array}{l}\text { Currency } \\
\text { pair }\end{array}$} & \multicolumn{2}{|c|}{ TSFDC-down } & \multicolumn{2}{c|}{ TSFDC-up } \\
\cline { 2 - 5 } EUR/CHF & Sharpe ratio & Sortino ratio & Sharpe ratio & Sortino ratio \\
\hline GBP/CHF & 2.6 & $\infty$ & 1.8 & $\infty$ \\
\hline EUR/USD & 3.2 & $\infty$ & 2.0 & $\infty$ \\
\hline GBP/AUD & 3.0 & 177.3 & 1.7 & $\infty$ \\
\hline GBP/JPY & 1.1 & $\infty$ & 3.4 & $\infty$ \\
\hline NZD/JPY & 2.7 & 37.2 & 0.9 & $\infty$ \\
\hline AUD/JPY & 2.6 & $\infty$ & 3.6 & $\infty$ \\
\hline EUR/NZD & 2.0 & $\infty$ & 4.2 & $\infty$ \\
\hline
\end{tabular}

Table 10: Summary of the monthly RR (\%) obtained by applying the buy and hold (B\&H) approach to each of the eight considered currency pairs. The trading period is from $1 / 1 / 2015$ to $31 / 7 / 2015$.

\begin{tabular}{|l|c|c|c|c|c|c|c|c|}
\hline & Jan & Feb & Mar & Apr & May & Jun & Jul & Sum \\
\hline EUR/CHF & -12.88 & 1.75 & -1.95 & 0.10 & -1.41 & 0.99 & 1.77 & -11.63 \\
\hline GBP/CHF & -9.68 & 5.17 & -2.01 & -0.60 & 0.57 & 1.92 & 2.69 & -1.94 \\
\hline EUR/USD & -6.48 & -1.07 & -3.66 & 3.96 & -2.31 & 1.72 & -1.38 & -9.22 \\
\hline GBP/AUD & 2.07 & 1.57 & -1.42 & -0.12 & 2.81 & 1.59 & 5.11 & 11.61 \\
\hline GBP/JPY & 5.43 & 4.59 & -3.73 & 3.34 & 3.32 & 1.34 & 0.81 & 4.24 \\
\hline NZD/JPY & -9.04 & 6.60 & -1.14 & 1.60 & -2.93 & -5.41 & -1.84 & -12.16 \\
\hline AUD/JPY & -7.28 & 3.02 & -2.26 & 3.49 & 0.49 & 0.27 & 4.48 & 2.21 \\
\hline EUR/NZD & 0.54 & -5.08 & -2.54 & 2.38 & 4.43 & 6.12 & 1.79 & 7.64 \\
\hline
\end{tabular}

\section{Experiment 1: Results' Discussion}

We begin with an examination of the results obtained from the buy-and-hold strategy (shown in Table 10). For each currency pair, we note that the buy and hold approach does generate profit in some months, but incurs losses in others. This observation indicates that none of the selected currency pairs exhibit a monotonic trend during the trading period. Besides, the numbers shown in the 'Sum' column (Table 10) show that the B\&H method generates profit in four cases: GBP/AUD, GBP/JPY, AUD/JPY, and EUR/NZD (with rate of returns $R R$ of up to $11.61 \%$ in the case of GBP/AUD). The same column also shows that the buy and hold method incurs losses in the other four cases (with $R R$ equal to -12.16 in the case of NZD/JPY). These observations support our claim regarding the variation of trends of the selected currency rates in Section 5.1.

We then examine the profitability of both versions of TSFDC. The monthly rate of returns $(R R)$ reported in Tables 7 and 8 show that both versions of TSFDC are mostly profitable (except in very 
few cases; e.g. trading with TSFDC-down on EUR/USD in March 2015 when it incurred losses of $0.36 \%$, Table 7). The results in column (RR\%), shown in Table 6 , suggests that TSFDC can be highly profitable (with rate of return, $R R$, of up to $571.89 \%$, as in the case of applying TSFDC-up to EUR/NZD - last row in Table 6). The overall Win Ratio of TSFDC (i.e. the probability of having a winning trade) ranges between 0.77 (as in the case of applying TSFDC-down to EUR/NZD) and 0.65 (in the case of applying TSFDC-down to EUR/USD). We consider this range to be reasonably acceptable.

However, it is important to note that the profitability of TSFDC can vary largely from one currency pair to another - as demonstrated in Table 6 when TSFDC-up is applied to GBP/JPY and EUR/NZD. One can easily observe an important difference between the produced total $R R$ (from $28.91 \%$ for GBP/JPY, compared to $571.89 \%$ for EUR/NZD). Likewise, in the same table, other evaluation metrics (e.g. profit factor and Win Ratio) reveal a better performance for TSFDC in the case of EUR/NZD than on the other pairs. This indicates that, whilst TSFDC may generate profits in most cases, its performance may vary substantially from one currency rate to another. It follows then that a trader may want to consider other currency pairs as TSFDC may, possibly, perform better on these currencies than on those reported in this paper.

When we inspect the risk of TSFDC, in Table 6, we notice that, in most cases, the maximum drawdown $(M D D)$ is no worse than - 6.0\% (except in two cases: EUR/CHF and GBP/CHF) - values we consider to be relatively low. Moreover, the values of the Sortino ratio, reported in Table 9, are, in most cases, a positive infinity ( $\infty)$. This reflects the fact that the downside risk (see (10) in Section 5.3) of TSFDC is null in most of these experiments.

Lastly, we examine the risk-adjusted performance of TSFDC. For this purpose, we consider the values of the Sharpe ratio Table 9. We note that TSFDC provides Sharpe ratio consistently. A positive Sharpe ratio indicates that the TSFDC has surpassed the 5\% annual risk-free rate, demonstrating that TSFDC generates worthy excess returns for each additional unit of risk it takes. We conclude that TSFDC earns more than enough return to compensate for the risk it took over the trading period.

We conclude from the previous analysis that TSFDC-down and TSFDC-up generate more returns than the buy and hold method. Additionally, both versions of TSFDC can be highly profitable, with $R R$ of more than $400 \%$ (Table 6). We also showed that TSFDC consistently delivers a positive Sharpe ratio. Finally, the established variety of the selected currency pairs in the initial dataset (Section 5.1) support our objective that TSFDC can be profitably applied to a wide range of currency rates.

\subsection{Experiment 2: Compare the return and risk of both versions of TSFDC}

The objective of this experiment is to test whether there is a significant difference between the return and risk of both versions of TSFDC, TSFDC-up and TSFDC-down. We consider the monthly rate of returns $(R R)$ and monthly maximum drawdown $(M D D)$. We use the Wilcoxon signed rank test to validate our conclusion statistically.

Firstly, we apply the Wilcoxon test with the null hypothesis that the two sets of monthly $R R$ of TSFDC-down and TSFDC-up are not different. Each of these two sets consists of 56 observations. Appendix A comprises the details of these two sets. In this case, the Wilcoxon test returns a $p$-value of 0.79. Since the $p$-value is greater than 0.05 , the Wilcoxon test cannot reject the null hypothesis that there is no difference between the monthly $R R$ for TSFDC-down and TSFDC-up.

Secondly, we apply the Wilcoxon test with the null hypothesis being that there is no difference between the two sets of monthly $M D D$ of TSFDC-down and TSFDC-up. Appendix A compiles the details of these two sets. In this case, the Wilcoxon test returned a $p$-value of 0.50 . This $p$-value is greater than 0.05 . Therefore, the Wilcoxon test cannot reject the null hypothesis that there is no difference between the two sets of monthly $M D D$ for TSFDC-down and TSFDC-up. To conclude, 
Wilcoxon tests do not suggest that the monthly returns and the risk (measured as $M D D$ ) of TSFDCdown and TSFDC-up are different.

\section{COMPARING TSFDC TO ANOTHER DC-BASED STRATEGY: THE 'DC+GA'}

In this section, we compare TSFDC with the trading strategy named ' $\mathrm{DC}+\mathrm{GA}$ ' established by Kampouridis and Otero [21]. DC+GA runs $N_{\text {theta }}$ DC summaries concurrently (using $N_{\text {theta }}$ thresholds; where $N_{\text {theta }}$ is a parameter to be chosen by the investor). Each DC summary is associated with particular values of some trading parameters (see Table 1, page 151, [21]). Each DC summary analyze the current price and uses its trading parameters to generate a buy or sell recommendation. Each DC summary is given a 'weight' based on the profitability of its established recommendations. The $N_{\text {theta }}$ DC-thresholds produce $N_{\text {theta }}$ recommendations. These thresholds are, then, clustered in two groups based on the proposed recommendations: the first group comprises the thresholds those recommend a buy action, the second group comprises those recommending a sell action.

To make a buy or sell decision, DC+GA sum the weights of the thresholds belongs to each group (i.e. cluster): if the sum of the weights for all thresholds recommending a buy (sell) action is greater than the sum of the weights for all thresholds recommending a sell (buy) action, then the strategy's action will be to buy (sell).

DC+GA applies a Genetic Algorithm (GA) model to optimize the trading parameters' values for each DC summary. The output of the GA is a set of $N_{\text {theta }}$ DC-thresholds, each of which being associated with a 'weight'. The evolution of the GA consists of finding the best set of DC thresholds along with their trading parameters and weights that maximize the total profits. The best set of DC's thresholds, and their associated weight and trading parameters, will be used for trading during the out-of-sample trading period [21].

We identify the following differences between TSFDC and DC + GA:

- In contrast to DC + GA, TSFDC is a counter trend strategy.

- In contrast to DC + GA, TSFDC is based on a forecasting model established under the DC context.

- TSFDC uses exactly two thresholds for DC summary (STheta and BTheta), whereas DC+GA relies on $N_{\text {theta }}$ DC summaries.

Kampouridis and Otero [21] report the average monthly returns of applying DC+GA to five currency pairs (shown in Table 6 [21], page 158). We note that DC+GA incurs overall losses in two out of the five cases. Moreover, when examining the reported monthly returns (see Tables 5 and A1, pages 156 and 158 respectively, [21]) one can easily note that the proposed trading models incur losses in about $50 \%$ of the cases! The authors conclude that the proposed model "...could not consistently return profitable strategies and thus their mean returns were negative." By contrast, when inspecting the monthly returns of TSFDC reported in Tables 7 and 8, we note that in the majority of cases TSFDC's monthly returns are positive. Furthermore, the overall returns of applying TSFDC to the eight currency pairs (over the trading period of seven months) are consistently positive (see Table 6, Section 7.1). Thus, we conclude that TSFDC is more profitable than DC+GA.

We then examine the risk-adjusted returns of DC+GA and DBA. The authors in [21] do not provide any risk-adjusted measurement for DC+GA. However, based on the reported monthly returns in Table 5 (Kampouridis and Otero [21], page 158), we can compute the Sharpe ratio. If we consider a risk-free rate of 5\% per annum, then we find that DC+GA will have negative Sharpe ratio in four out of the five considered currency pairs as follow:

- $\quad$ In the case of EUR/GBP: - 0.9 
- $\quad$ In the case of EUR/JPY: 0.2

- $\quad$ In the case of EUR/USD: -0.7

- In the case of GBP/CHF: - 0.6

- In the case of GBP/USD: - 0.1

Whereas, TSFDC consistently produces a positive Sharpe ratio (see Table 9). Based on this analysis, we conclude that TSFDC outperforms "DC+GA" in terms of profitability and risk-adjusted returns. To conclude, by comparing the results of DC+ GA ( [21]) and the results of TSFDC (Section 7.1) we conclude that TSFDC outperforms DC+GA regarding produced returns and risk-adjusted returns.

Finally, we should note that the other DC-based strategies (e.g. [20] [22]) do not rely any forecasting models. To the best of our knowledge, TSFDC is the first DC-based trading strategy that is based on a clearly articulated forecasting approach.

\section{SUMMARY AND CONCLUSION}

The Directional Changes Framework (DC) framework segments the market into alternating downtrends and uptrends. The majority of existing trading strategies provide trading rules based on time series. Very few trading models were developed under the DC framework.In this paper, our objective is to develop a successful trading strategy based on forecasting DC. To this end, we use the forecasting model presented in Bakhach et al., [11] to develop a trading strategy named TSFDC. TSFDC is a contrarian trading strategy that relies on the forecasting model, summarized in Section 3 , to decide when to generate a buy or sell signal.

The performance of TSFDC was examined using eight currency pairs. We utilized 1-minute trade records for these eight currency rates covering the period between 1/1/2013 and 31/7/2015. We chose these currency pairs such that they exhibited various trends during the considered trading period of seven months (Section 5.4). We trained and tested the TSFDC model using a monthly-basis rolling window approach. Each rolling window comprised 1) a training period, used to train the forecasting model developed in Bakhach et al. [11] (24 months in length), and 2) a trading period (1 month in length) to which we applied TSFDC (Section 5.4). We used a set of evaluation metrics to assess the performance of TSFDC.

By examining the rate of returns reported in Table 6 (Section 7.1), we can conclude that TSFDC can be highly profitable (with a rate of return, $R R$, of more than $500 \%$, as per EUR/NZD) and yet still have an acceptable level of risk (with $M D D$ equal to $-5.1 \%$ ). The results in Table 6 show that the performance of TSFDC can vary substantially from one currency pair to another. We also argued that TSFDC outperforms another DC-based trading strategy in Section 8.

As our main contribution, we proved that TSFDC outperforms the buy-and-hold approach in terms of produced returns. We showed that TSFDC outperforms another DC-based trading strategy (Section 6.7). We demonstrated that TSFDC can be highly profitable. We also showed that TSFDC consistently delivers a positive Sharpe ratio. We demonstrated the effectiveness of TSFDC over eight different currency rates. Therefore, we believe that TSFDC is feasible in a broad range of currencies (since these eight currency pairs have different patterns).

In future works, we should examine the impact of other factors, such as slippage cost and bid-ask spread, on the performance of TSFDC for more realistic estimation. The incorporation of an intelligent money management approach should improve the performance of TSFDC. 


\section{References}

[1] H. Ao and E. Tsang, "Capturing Market Movements with Directional Changes," Working Paper WP069-13, Centre for Computational Finance \& Economic Agents (CCFEA), University of Essex, Colchester, 2013.

[2] M. Aloud, M. Fasli, E. Tsang, A. Dupuis and R. Olsen, "Stylized Facts of the FX Market Transactions Data: An Empirical Study," Journal of Finance and Investment Analysis, vol. 2, pp. 145-183, 2013.

[3] J. Glattfelder, A. Dupuis and R. Olsen, "Patterns in high-frequency FX data: Discovery of 12 empirical scaling laws," Quantitative Finance, vol. 11, no. 4, pp. 599-614, 2011.

[4] S. Masry, "Event-Based Microscopic Analysis of the FX Market," Ph.D. thesis, Centre for Computational Finance \& Economic Agents (CCFEA), University of Essex, Colchester, 2013.

[5] M. Aloud, E. K. Tsang and R. Olsen, "Modelling the FX market traders' behaviour: an agentbased approach," in Simulation in Computational Finance and Economics: Tools and Emerging Applications, B. Alexandrova-Kabadjova, S. Martinez-Jaramillo, A. Garcia-Almanza and E. Tsang, Eds., Hershey, PA, IGI Global, 2012.

[6] X. Li, Z. Deng and J. Luo, "Trading strategy design in financial investment through a turning points prediction scheme," Expert System With Application, vol. 36, pp. 7818-7826, 2009.

[7] T. L. Chen and F. Y. Chen, "An intelligent pattern recognition model for supporting investment decisions in stock market," International Journal of Information Sciences, vol. 346, pp. 261274, 2016.

[8] M. Göçkena, M. Özçalıcıb, A. Borua and A. T. Dosdogru, "Integrating metaheuristics and Artificial Neural Networks for improved stock price prediction," Expert Systems With Applications, vol. 44, p. 320-331, 2016.

[9] S. Galeshchuk and S. Mukherjee, "Deep networks for predicting direction of change in foreign exchange rates," Intelligent Systems in Accounting, Finance and Management, 19 March 2017.

[10] J. T. Davis and A. Episcopos, "Predicting direction shifts on Canadian-US exchange rates with artificial neural networks," Intelligent Systems in Accounting, Finance \& Management, vol. 10, no. 2, pp. 83-96, 2001.

[11] A. Bakhach, E. P. Tsang and H. Jalalian, "Forecasting Directional Changes in the FX Markets," in IEEE Symposium on Computational Intelligence for Financial Engineer \& Economic (CIFEr' 2016), Athens, Greece,, 2016.

[12] R. C. Cavalcante, R. C. Brasileiro, V. L. Souza, J. P. Nobrega and A. L. Oliveira, "Computational intelligence and financial markets: A survey and future directions," Expert Systems with Applications, vol. 55, pp. 194-211, 2016.

[13] D. Guillaume, M. Dacorogna, R. Davé, U. Müller, R. Olsen and O. Pictet, "From the bird's eye to the microscope: A survey of new stylized facts of the intra-daily foreign exchange markets," Finance and stochastic, vol. 1, no. 2, pp. 95-129, 1997. 
[14] E. P. K. Tsang, R. Tao and S. Ma, "Profiling Financial Market Dynamics under Directional Changes," Quantitative finance, vol. 17, no. 2, pp. 217-225, 2017.

[15] A. Azzini, C. d. C. Pereira and A. G. Tettamanzi, "Predicting Turning Points in Financial Markets with Fuzzy-Evolutionary and Neuro-Evolutionary Modeling," in Applications of Evolutionary Computing, Lecture Notes in Computer Science, vol 5484. , Berlin, Heidelberg, Springer-Verlag, 2009, pp. 213-222.

[16] S. Raftopoulos, "The Zigzag Trend Indicator," Technical Analysis of Stocks and Commodities, vol. 21, no. 11, p. 26-32, 2003.

[17] T. Bisig, A. Dupuis, V. Impagliazzo and R. Olsen, "The scale of market quake," Quantitative Finance, vol. 12, no. 4, pp. 501-508, 2012.

[18] S. Masry and E. Tsang, "Simulating market clearance dynamics under a simple event calculus market model," in 3rd Computer Science and Electronic Engineering Conference, Colchester, UK, 2011.

[19] A. Golub, G. Chliamovitch, A. Dupuis and B. Chopard, "Multi-Scale Representation of High Frequency Market Liquidity," SSRN, 10 Feb. 2014. [Online]. Available: http://papers.ssrn.com/sol3/papers.cfm?abstract_id=2393428. [Accessed 26 Jun. 2016].

[20] A. Bakhach, E. P. K. Tsang, W. L. Ng and V. L. R. Chinthalapati, "Backlash Agent: A trading strategy based on Directional Change," in IEEE Symposium on Computational Intelligence for Financial Engineer \& Economic (CIFEr' 2016), Athens, Greece,, 2016.

[21] M. Kampouridis and F. E. Otero, "Evolving trading strategies using directional changes," Expert Systems With Applications, vol. 73, pp. 145-160, 2017.

[22] A. Golub, J. B. Glattfelder and R. B. Olsen, "The Alpha Engine: Designing an Automated Trading Algorithm," in High-Performance Computing in Finance: Problems, Methods, and Solutions, CRC Press, 2017.

[23] R. Pardo, The evalution and optimization of trading strategy, NJ, USA: John Wiley \& Sons, 2011.

[24] M. Kearns, A. Kulesza and Y. Nevmyvaka, "Empirical limitations on High-Frequency Trading Profitability," Journal of Trading, vol. 5, no. 4, p. 50-62, 2010.

[25] E. de Faria, M. P. Albuquerque, J. Gonzalez, J. Cavalcante and M. P. Albuquerque, "Predicting the Brazilian stock market through neural networks and adaptive exponential smoothing methods," Expert Systems with Applications, vol. 36, no. 10, p. 12506-12509, 2009.

[26] B. Krollner, B. Vanstone and G. Finnie, "Financial time series forecasting with machine learning techniques: A survey," in European Symposium on Artificial Neural Networks: Computational and Machine Learning, Bruges, Belgium, 2010.

[27] I. Aldridge, High-Frequency Trading, New Jersey, USA.: John Wiley \& Sons, Wiley Trading series, 2013.

[28] V. Vella and W. L. Ng, "A Dynamic Fuzzy Money Management Approach for Controlling the Intraday Risk-Adjusted Performance of AI Trading Algorithms," Intelligent Systems in Accounting, Finance and Management, vol. 22, no. 2, pp. 153-178, 2015. 
[29] W. F. Sharpe, "Asset allocation: Management Style and Performance Measurement," Journal of Portfolio Management, vol. Winter, pp. 7-19, 1992.

[30] J. Prokop, "Further evidence on the role of ratio choice in hedge fund performance evaluation," Journal of Finance and Investment Analysis, vol. 1, no. 3, p. 181-195, 2012.

[31] B. R. Auer and F. Schuhmacher, "Robust evidence on the similarity of sharpe ratio and drawdown-based hedge fund performance rankings," Journal of international financial markets, institutions and money, vol. 24, pp. 153-165, 2013.

[32] J. L. Treynor, "How to rate management of investment funds," Harvard Business Review, vol. 43, no. 1 , pp. 63-75, 1965.

[33] F. Sortino and R. van der Meer, "Downside risk," Journal of Portfolio Management, vol. 17, no. 4, pp. 27-31, 1991.

[34] M. Qi and Y. Wu, "Technical trading-rule profitability, data snooping, and reality check: Evidence from the foreign exchange market," Journal of Money, Credit and Banking, vol. 38, pp. 2135-2158, 2006.

[35] P.-H. Hsu and M. P. Taylor, "Forty Years, Thirty Currencies and 21,000 Trading Rules: A Large-Scale, Data-Snooping Robust Analysis of Technical Trading in the Foreign Exchange Market," 5 May 2013. [Online]. Available: Available at SSRN: https://ssrn.com/abstract=2517125. [Accessed 30 June 2017].

[36] N. Zarrabia, S. Snaithb and J. Coakleya, "FX technical trading rules can be profitable sometimes!," International Review of Financial Analysis, vol. 49, p. 113-127, 2017.

[37] S. Bohn, "The slippage paradox," 14 March 2011. [Online]. Available: http://arxiv.org/pdf/1103.2214. [Accessed 22 June 2017].

[38] F. Wilcoxon., " Individual comparisons by ranking methods," Biometrics Bulletin, vol. 1, pp. 80-83, 1945. 


\section{Appendix A: Comparing the Return and Risk of TSFDC-down and TSFDC-up}

In Experiment 2, we aimed to test whether the TSFDC-down and TSFDC-up has different profitability and risk. The profitability is measured as monthly rate of returns $(R R)$. The risk is measured as $M D D$. In the following table we summarize the results of monthly $R R$ and $M D D$ obtained by applying TSFDC-down and TSFDC-up to the eight currency pairs based on the experiments conducted in Section 6.3. As can be noted in Table A1 below, we have two sets of monthly $R R$ : one for TSFDC-down and the other is for TSFDC-up. Each set encompass 56 observations. We apply the non-parametric Wilcoxon test with the null hypothesis being that these two sets are not different.

As can be noted in Table A.1 below, we have two sets of monthly $R R$ under the column 'RR(\%)': one for TSFDC-down and the other is for TSFDC-up. Each set encompass 56 observations. We apply the non-parametric Wilcoxon test with the null hypothesis being that these two sets are not different.

Similarly, Table A.1 identifies two sets of monthly $M D D$ under the column 'MDD(\%)': one for TSFDC-down and the other is for TSFDC-up. Each set encompass 56 observations. We apply the non-parametric Wilcoxon test with the null hypothesis being that these two sets are not different.

Table A.1: Summary of monthly rate of returns $(R R)$ and $M D D$ of TSFDC-down and TSFDC-up based on Experiment 2 (Section 7.2)

\begin{tabular}{|c|c|c|c|c|c|c|}
\hline \multirow{2}{*}{$\begin{array}{c}\text { Observation } \\
\text { number }\end{array}$} & \multirow{2}{*}{$\begin{array}{l}\text { Currency } \\
\text { pairs }\end{array}$} & \multirow{2}{*}{$\begin{array}{l}\text { Trading } \\
\text { Month }\end{array}$} & \multicolumn{2}{|c|}{ RR (\%) } & \multicolumn{2}{|c|}{ MDD (\%) } \\
\hline & & & $\begin{array}{l}\text { TSFDC- } \\
\text { down }\end{array}$ & $\begin{array}{l}\text { TSFDC- } \\
\text { up }\end{array}$ & $\begin{array}{l}\text { TSFDC- } \\
\text { down }\end{array}$ & $\begin{array}{l}\text { TSFDC- } \\
\text { up }\end{array}$ \\
\hline 1 & \multirow{7}{*}{ 卢罝 } & Jan & 4.47 & 4.26 & -13.4 & -15.1 \\
\hline 2 & & Feb & 14.40 & 9.75 & -1.4 & -2.5 \\
\hline 3 & & Mar & 17.59 & 16.87 & -0.6 & -3.4 \\
\hline 4 & & Apr & 7.58 & 5.71 & -0.7 & -2.8 \\
\hline 5 & & May & 13.37 & 7.61 & -0.7 & -1.5 \\
\hline 6 & & Jun & 12.41 & 10.15 & -1.4 & -3.3 \\
\hline 7 & & Jul & 14.77 & 8.68 & -0.6 & -1.8 \\
\hline 8 & \multirow{7}{*}{$\frac{1}{\text { 岂 }}$} & Jan & 13.59 & 31.54 & -12.1 & -10.8 \\
\hline 9 & & Feb & 19.02 & 16.30 & -2.7 & -3.9 \\
\hline 10 & & Mar & 14.96 & 21.67 & -2.9 & -3.8 \\
\hline 11 & & Apr & 6.71 & 12.34 & -2.5 & -4.0 \\
\hline 12 & & May & 9.85 & 7.59 & -2.9 & -3.1 \\
\hline 13 & & Jun & 15.17 & 14.13 & -3.7 & -4.1 \\
\hline 14 & & Jul & 14.73 & 11.62 & -2.2 & -2.8 \\
\hline 15 & \multirow{7}{*}{$\underset{\text { 总 }}{\stackrel{2}{2}}$} & Jan & 1.12 & 6.81 & -4.2 & -5.8 \\
\hline 16 & & Feb & 7.54 & 9.27 & -3.1 & -3.9 \\
\hline 17 & & Mar & -0.36 & 1.69 & -5.0 & -4.8 \\
\hline 18 & & Apr & 4.20 & 1.66 & -2.9 & -3.9 \\
\hline 19 & & May & 5.73 & 9.67 & -3.3 & -2.5 \\
\hline 20 & & Jun & 7.85 & 6.13 & -3.7 & -2.8 \\
\hline 21 & & Jul & 0.96 & 0.86 & -3.4 & -3.0 \\
\hline
\end{tabular}


Table A.1 (continued): summary of monthly rate of returns $(R R)$ and $M D D$ of TSFDC-down and TSFDC-up based on Experiment 2.

\begin{tabular}{|c|c|c|c|c|c|c|}
\hline \multirow{2}{*}{$\begin{array}{c}\text { Observation } \\
\text { number }\end{array}$} & \multirow{2}{*}{$\begin{array}{c}\text { Currency } \\
\text { pairs }\end{array}$} & \multirow{2}{*}{$\begin{array}{l}\text { Trading } \\
\text { Month }\end{array}$} & \multicolumn{2}{|c|}{ RR (\%) } & \multicolumn{2}{|c|}{ MDD } \\
\hline & & & $\begin{array}{l}\text { TSFDC- } \\
\text { down }\end{array}$ & $\begin{array}{c}\text { TSFDC- } \\
\text { up }\end{array}$ & $\begin{array}{l}\text { TSFDC- } \\
\text { down }\end{array}$ & $\begin{array}{c}\text { TSFDC- } \\
\text { up }\end{array}$ \\
\hline 22 & \multirow{7}{*}{$\frac{3}{3}$} & Jan & 19.70 & 13.34 & -2.83 & -1.36 \\
\hline 23 & & Feb & 10.51 & 10.06 & -3.18 & -3.52 \\
\hline 24 & & Mar & 10.14 & 9.09 & -1.53 & -1.56 \\
\hline 25 & & Apr & 13.52 & 9.23 & -1.14 & -2.39 \\
\hline 26 & & May & 15.97 & 9.51 & -0.84 & -1.39 \\
\hline 27 & & Jun & 11.52 & 5.97 & -1.25 & -1.29 \\
\hline 28 & & Jul & 11.27 & 5.83 & -3.35 & -1.91 \\
\hline 29 & \multirow{7}{*}{ 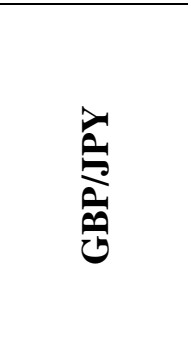 } & Jan & 7.72 & 11.39 & -4.8 & -4.2 \\
\hline 30 & & Feb & 6.40 & 3.64 & -3.8 & -3.2 \\
\hline 31 & & Mar & 4.04 & 6.00 & -2.8 & -5.7 \\
\hline 32 & & Apr & 7.05 & 3.07 & -4.7 & -2.9 \\
\hline 33 & & May & 8.38 & 4.11 & -3.5 & -1.9 \\
\hline 34 & & Jun & 0.99 & 4.16 & -4.1 & -3.0 \\
\hline 35 & & Jul & -2.10 & -3.46 & -3.1 & -3.7 \\
\hline 36 & \multirow{7}{*}{ 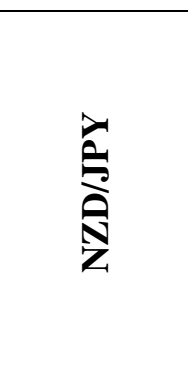 } & Jan & 19.14 & 26.96 & -2.6 & -4.0 \\
\hline 37 & & Feb & 26.90 & 18.06 & -3.2 & -3.0 \\
\hline 38 & & Mar & 19.95 & 24.06 & -4.9 & -2.2 \\
\hline 39 & & Apr & 30.41 & 22.98 & -2.8 & -2.9 \\
\hline 40 & & May & 24.27 & 24.92 & -3.1 & -2.4 \\
\hline 41 & & Jun & 17.20 & 32.66 & -2.6 & -3.0 \\
\hline 42 & & Jul & 45.26 & 41.09 & -3.1 & -2.2 \\
\hline 43 & \multirow{7}{*}{ 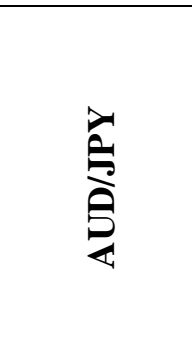 } & Jan & 15.36 & 21.48 & -5.0 & -2.3 \\
\hline 44 & & Feb & 16.47 & 14.88 & -3.2 & -2.3 \\
\hline 45 & & Mar & 10.51 & 17.30 & -2.9 & -4.2 \\
\hline 46 & & Apr & 16.69 & 12.25 & -2.8 & -5.2 \\
\hline 47 & & May & 25.51 & 21.15 & -2.1 & -2.6 \\
\hline 48 & & Jun & 10.48 & 17.32 & -3.6 & -3.5 \\
\hline 49 & & Jul & 9.09 & 11.97 & -3.1 & -3.1 \\
\hline 50 & \multirow{7}{*}{$\underset{\text { 空 }}{\stackrel{\mathbf{z}}{\mathbf{z}}}$} & Jan & 24.12 & 26.27 & -1.2 & -5.1 \\
\hline 51 & & Feb & 50.04 & 68.74 & -4.6 & -2.7 \\
\hline 52 & & Mar & 49.76 & 64.56 & -2.1 & -3.9 \\
\hline 53 & & Apr & 59.39 & 78.72 & -2.8 & -1.9 \\
\hline 54 & & May & 79.92 & 82.81 & -3.0 & -2.9 \\
\hline 55 & & Jun & 104.91 & 101.88 & -2.8 & -2.9 \\
\hline 56 & & Jul & 120.99 & 148.91 & -2.9 & -2.6 \\
\hline
\end{tabular}

